REPRESENTATION THEORY

An Electronic Journal of the American Mathematical Society

Volume 7, Pages 651-680 (November 24, 2003)

S $1088-4165(03) 00211-5$

\title{
A GEOMETRIC APPROACH TO STANDARD MONOMIAL THEORY
}

\author{
M. BRION AND V. LAKSHMIBAI
}

\begin{abstract}
We obtain a geometric construction of a "standard monomial basis" for the homogeneous coordinate ring associated with any ample line bundle on any flag variety. This basis is compatible with Schubert varieties, opposite Schubert varieties, and unions of intersections of these varieties. Our approach relies on vanishing theorems and a degeneration of the diagonal; it also yields a standard monomial basis for the multi-homogeneous coordinate rings of flag varieties of classical type.
\end{abstract}

\section{INTRODUCTION}

Consider the Grassmannian $X$ of linear subspaces of dimension $r$ in $k^{n}$, where $k$ is a field. We regard $X$ as a closed subvariety of projective space $\mathbb{P}\left(\wedge^{r} k^{n}\right)$ via the Plücker embedding; let $L$ be the corresponding very ample line bundle on $X$. Then the ring $\bigoplus_{m=0}^{\infty} H^{0}\left(X, L^{\otimes m}\right)$ admits a nice basis, defined as follows.

Let $\left\{v_{1}, \ldots, v_{n}\right\}$ be the usual basis of $k^{n}$; then the $v_{i_{1}} \wedge \cdots \wedge v_{i_{r}}, 1 \leq i_{1}<\cdots<$ $i_{r} \leq n$, form a basis of $\wedge^{r} k^{n}$. We put $I=\left(i_{1}, \ldots, i_{r}\right), v_{I}=v_{i_{1}} \wedge \cdots \wedge v_{i_{r}}$, and we denote by $\left\{p_{I}\right\}$ the dual basis of the basis $\left\{v_{I}\right\}$; the $p_{I}$ (regarded in $H^{0}(X, L)$ ) are the Plücker coordinates. Define a partial order on the set $\mathcal{I}$ of indices $I$ by letting $I=\left(i_{1}, \ldots, i_{r}\right) \leq\left(j_{1}, \ldots, j_{r}\right)=J$ if and only if $i_{1} \leq j_{1}, \ldots, i_{r} \leq j_{r}$. Then

(i) The monomials $p_{I_{1}} p_{I_{2}} \cdots p_{I_{m}}$ where $I_{1}, \ldots, I_{m} \in \mathcal{I}$ satisfy $I_{1} \leq I_{2} \leq \cdots \leq I_{m}$, form a basis of $H^{0}\left(X, L^{\otimes m}\right)$.

(ii) For any $I, J \in \mathcal{I}$, we have $p_{I} p_{J}-\sum_{I^{\prime}, J^{\prime}, I^{\prime} \leq I, J \leq J^{\prime}} a_{I^{\prime} J^{\prime}} p_{I^{\prime}} p_{J^{\prime}}=0$, where $a_{I^{\prime} J^{\prime}} \in k$.

The monomials in (i) are called the standard monomials of degree $m$, and the relations in (ii) are the quadratic straightening relations; they allow us to express any nonstandard monomial in the $p_{I}$ as a linear combination of standard monomials.

Further, this standard monomial basis of the homogeneous coordinate ring of $X$ is compatible with its Schubert subvarieties, in the following sense. For any $I \in \mathcal{I}$, let $X_{I}=\left\{V \in X \mid \operatorname{dim}\left(V \cap \operatorname{span}\left(v_{1}, \ldots, v_{s}\right)\right) \geq \#\left(j, i_{j} \leq s\right), 1 \leq s \leq r\right\}$ be the corresponding Schubert variety; then the restriction $\left.p_{J}\right|_{X_{I}}$ is nonzero if and only if $J \leq I$. The monomial $p_{I_{1}} \cdots p_{I_{m}}$ will be called standard on $X_{I}$ if $I_{1} \leq \cdots \leq I_{m} \leq I$; equivalently, this monomial is standard and does not vanish identically on $X_{I}$. Now:

(iii) The standard monomials of degree $m$ on $X_{I}$ restrict to a basis of $H^{0}\left(X_{I}, L^{\otimes m}\right)$. The standard monomials of degree $m$ that are not standard on $X_{I}$, form a basis of the kernel of the restriction map $H^{0}\left(X, L^{\otimes m}\right) \rightarrow H^{0}\left(X_{I}, L^{\otimes m}\right)$.

Received by the editors November 8, 2001 and, in revised form, September 12, 2003.

2000 Mathematics Subject Classification. Primary 14M15, 20G05, 14L30, 14L40. 
These classical results go back to Hodge; see [5]. They have important geometric consequences, e.g., $X$ is projectively normal in the Plücker embedding; its homogeneous ideal is generated by the quadratic straightening relations; the homogeneous ideal of any Schubert variety $X_{I}$ is generated by these relations together with the $p_{J}$ where $J \not \leq I$.

The purpose of Standard Monomial Theory (SMT) is to generalize Hodge's results to any flag variety $X=G / P$ (where $G$ is a semisimple algebraic group over an algebraically closed field $k$, and $P$ a parabolic subgroup) and to any effective line bundle $L$ on $X$. SMT was developed by Lakshmibai, Musili, and Seshadri in a series of papers, culminating in [9] where it is established for all classical groups $G$. There the approach goes by ascending induction on the Schubert varieties, using their partial resolutions as projective line bundles over smaller Schubert varieties.

Further results concerning certain exceptional or Kac-Moody groups led to conjectural formulations of a general SMT; see [10. These conjectures were then proved by Littelmann, who introduced new combinatorial and algebraic tools: the path model of representations of any Kac-Moody group, and Lusztig's Frobenius map for quantum groups at roots of unity (see [11, 12]).

In the present paper, we obtain a geometric construction of a SMT basis for $H^{0}(X, L)$, where $X=G / P$ is any flag variety and $L$ is any ample line bundle on $X$. This basis is compatible with Schubert varieties (that is, with orbit closures in $X$ of a Borel subgroup $B$ of $G$ ) and also with opposite Schubert varieties (the orbit closures of an opposite Borel subgroup $B^{-}$); in fact, it is compatible with any intersection of a Schubert variety with an opposite Schubert variety. We call such intersections Richardson varieties, since they were first considered by Richardson in [17]. Our approach adapts to the case where $L$ is an effective line bundle on a flag variety of classical type in the sense of [9]. This sharpens the results of [9] concerning the classical groups.

Our work may be regarded as one step towards a purely geometric proof of Littelmann's results concerning SMT. He constructed a basis of $T$-eigenvectors for $H^{0}(X, L)$ (where $T$ is the maximal torus common to $B$ and $B^{-}$) indexed by certain piecewise linear paths in the positive Weyl chamber, called $L S$ paths. This basis turns out to be compatible with Richardson varieties; notice that these are $T$-invariant. In fact, the endpoints of the path indexing a basis vector parametrize the smallest Richardson variety where this vector does not vanish identically (see [8]). If $L$ is associated with a weight of classical type, then the LS paths are just line segments: they are uniquely determined by their endpoints. This explains a posteriori why our geometric approach completes the program of SMT in that case.

In fact, our approach of SMT for an ample line bundle $L$ on a flag variety $X$ uses little of the rich geometry and combinatorics attached to $X$. Specifically, we only rely on vanishing theorems for unions of Richardson varieties (these being direct consequences of the existence of a Frobenius splitting of $X$, compatible with Schubert varieties and opposite Schubert varieties), together with the following property.

(iv) The diagonal in $X \times X$ admits a flat $T$-invariant degeneration to the union of all products $X_{w} \times X^{w}$, where the $X_{w}$ are the Schubert varieties and the $X^{w}$ are the corresponding opposite Schubert varieties.

The latter result follows from [2] (we provide a direct proof in Section 3). It plays an essential rôle in establishing generalizations of (i) and (iii); conversely, 
it turns out that the existence of a SMT basis implies (iv); see the Remark after Proposition 7.

It is worth noticing that (iv) is a stronger form of the fact that the classes of Schubert varieties form a free basis of the homology group (or Chow group) of $X$, the dual basis for the intersection pairing consisting of the classes of opposite Schubert varieties. This fact (in a different formulation) has been used by Knutson to establish an asymptotic version of the Littelmann character formula; see 7 .

This paper is organized as follows. In the preliminary Section 1, we introduce notation and study the geometry of Richardson varieties. Vanishing theorems for cohomology groups of line bundles on Richardson varieties are established in Section 2, by slight generalizations of the methods of Frobenius splitting. In Section 3, we construct filtrations of the $T$-module $H^{0}(X, L)$ that are compatible with restrictions to Richardson varieties. Our SMT basis of $H^{0}(X, L)$ is defined in Section 4; it is shown to be compatible with all unions of Richardson varieties. In Section 5 , we generalize statements (i) and (iii) above to any ample line bundle $L$ on a flag variety $G / P$; then (ii) follows from (i) together with compatibility properties of our basis. The case where the homogeneous line bundle $L$ is associated with a weight of classical type (e.g., a fundamental weight of a classical group) is considered in detail in Section 6. There we give a geometric characterization of the admissible pairs of [9] (these parametrize the weights of the $T$-module $H^{0}(X, L)$ ). The final Section 7 develops SMT for those effective line bundles that correspond to sums of weights of classical type.

Acknowledgements. The second author was partially supported by N.S.F. Grant DMS-9971295. She is grateful to the Institut Fourier for the hospitality extended during her visit in June 2001; it was there that this work originated.

\section{RICHARDSON VARIETIES}

The ground field $k$ is algebraically closed, of arbitrary characteristic. Let $G$ be a simply-connected semisimple algebraic group. Choose opposite Borel subgroups $B$ and $B^{-}$of $G$, with common torus $T$; let $\mathcal{X}(T)$ be the group of characters of $T$, also called weights. In the root system $R$ of $(G, T)$, we have the subset $R^{+}$of positive roots (that is, of roots of $(B, T)$ ), and the subset $S$ of simple roots. For each $\alpha \in R$, let $\check{\alpha}$ be the corresponding coroot and let $U_{\alpha}$ be the corresponding additive one-parameter subgroup of $G$, normalized by $T$.

We also have the Weyl group $W$ of $(G, T)$; for each $\alpha \in R$, we denote by $s_{\alpha} \in W$ the corresponding reflection. Then the group $W$ is generated by the simple reflections $s_{\alpha}, \alpha \in S$; this defines the length function $\ell$ and the Bruhat order $\leq$ on $W$. Let $w_{o}$ be the longest element of $W$, then $B^{-}=w_{o} B w_{o}$.

Let $P$ be a parabolic subgroup of $G$ containing $B$ and let $W_{P}$ be the Weyl group of $(P, T)$, a parabolic subgroup of $W$; let $w_{o, P}$ be the longest element of $W_{P}$. Each right $W_{P}$-coset in $W$ contains a unique element of minimal length; this defines the subset $W^{P}$ of minimal representatives of the quotient $W / W_{P}$. This subset is invariant under the map $w \longmapsto w_{o} w w_{o, P}$; the induced bijection of $W^{P}$ reverses the Bruhat order.

Each character $\lambda$ of $P$ defines a $G$-linearized line bundle on the homogeneous space $G / P$; we denote that line bundle by $L_{\lambda}$. The assignment $\lambda \longmapsto L_{\lambda}$ yields an isomorphism from the character group $\mathcal{X}(P)$ to the Picard group of $G / P$. Further, the line bundle $L_{\lambda}$ is generated by its global sections if and only if $\lambda$ (regarded as a 
character of $T)$ is dominant; in that case, $H^{0}\left(G / P, L_{\lambda}\right)$ is a $G$-module with lowest weight $-\lambda$.

Let $W_{\lambda}$ be the isotropy group of $\lambda$ in $W$, and let $P_{\lambda}$ be the parabolic subgroup of $G$ generated by $B$ and $W_{\lambda}$; then $W_{\lambda} \supseteq W_{P}, W^{\lambda} \subseteq W^{P}$, and $P_{\lambda} \supseteq P$. We shall identify $W^{\lambda}$ with the $W$-orbit of the weight $\lambda$, and denote by $w(\lambda)$ the image of $w \in W$ in $W / W_{\lambda} \simeq W^{\lambda}$.

The extremal weight vectors $p_{w(\lambda)} \in H^{0}\left(G / P, L_{\lambda}\right)$ are the $T$-eigenvectors of weight $-w(\lambda)$ for some $w \in W^{\lambda}$. These vectors are uniquely defined up to scalars.

We say that $\lambda$ is $P$-regular if $P_{\lambda}=P$. The ample line bundles on $G / P$ are the $L_{\lambda}$ where $\lambda$ is dominant and $P$-regular; under these assumptions, $L_{\lambda}$ is in fact very ample. We may then identify each $w \in W^{P}$ to $w(\lambda)$, and we put $p_{w}=p_{w(\lambda)}$.

The $T$-fixed points in $G / P$ are the $e_{w}=w P / P\left(w \in W / W_{P}\right)$; we index them by $W^{P}$. The $B$-orbit $C_{w}=B e_{w}$ is a Bruhat cell, an affine space of dimension $\ell(w)$; its closure in $G / P$ is the Schubert variety $X_{w}$. The complement $X_{w}-C_{w}$ is the boundary $\partial X_{w}$. We have

$$
\partial X_{w}=\bigcup_{v \in W^{P}, v<w} X_{v}
$$

and the irreducible components of $\partial X_{w}$ are the Schubert divisors $X_{v}$ where $v \in W^{P}$, $v<w$ and $\ell(v)=\ell(w)-1$. Then there exists $\beta \in R^{+}$such that $v=w s_{\beta}$.

Let $\lambda$ be a character of $P$ and let $f_{w}$ be the restriction to $X_{w}$ of the natural map $G / P \longrightarrow G / P_{\lambda}$; then $f_{w}\left(X_{w}\right)=X_{w(\lambda)}$. The set

$$
\partial_{\lambda} X_{w}:=f_{w}^{-1}\left(\partial X_{w(\lambda)}\right)
$$

is called the $\lambda$-boundary of $X_{w}$; it is the union of the Schubert divisors $X_{w s_{\beta}}$ where $\langle\lambda, \check{\beta}\rangle>0$. If $\lambda$ is dominant, then we have by Chevalley's formula:

$$
\operatorname{div}\left(\left.p_{w(\lambda)}\right|_{X_{w}}\right)=\sum\langle\lambda, \check{\beta}\rangle X_{w s_{\beta}}
$$

(sum over all $\beta \in R^{+}$such that $X_{w s_{\beta}}$ is a divisor in $X_{w}$ ). In particular, the zero set of $p_{w(\lambda)}$ in $X_{w}$ is $\partial_{\lambda} X_{w}$. If, in addition, $\lambda$ is $P$-regular, then $\partial_{\lambda} X_{w}=\partial X_{w}$.

We shall also need the opposite Bruhat cell $C^{w}=B^{-} e_{w}$ of codimension $\ell(w)$ in $G / P$, the opposite Schubert variety $X^{w}$ (the closure of $C^{w}$ ) and its boundary $\partial X^{w}$. Then $X^{w}=w_{o} X_{w_{o} w w_{o, P}}$ and

$$
\partial X^{w}=\bigcup_{v \in W^{P}, v>w} X^{v} .
$$

Recall that all Schubert varieties are normal and Cohen-Macaulay (thus, the same holds for all opposite Schubert varieties). Further, all scheme-theoretic intersections of unions of Schubert varieties and opposite Schubert varieties are reduced (see [14, 15, 16]).

Definition 1. Let $v, w$ in $W^{P}$. We call the intersection

$$
X_{w}^{v}:=X_{w} \cap X^{v}
$$

a Richardson variety in $G / P$. We define its boundaries by

$$
\left(\partial X_{w}\right)^{v}:=\partial X_{w} \cap X^{v} \text { and }\left(\partial X^{v}\right)_{w}:=X_{w} \cap \partial X^{v} \text {. }
$$

Notice that $X_{w}^{v}$ and its boundaries are closed reduced, $T$-stable subschemes of $G / P$. The $X_{w}^{v}$ were considered by Richardson, who showed, e.g., that they are 
irreducible (see [17]; the intersections $C_{w} \cap C^{v}$ were analyzed by Deodhar, see [4]). We shall give another proof of this result, and obtain a little more.

Lemma 1. (1) $X_{w}^{v}$ is nonempty if and only if $v \leq w$; then $X_{w}^{v}$ is irreducible of dimension $\ell(w)-\ell(v)$, and $\left(\partial X_{w}\right)^{v},\left(\partial X^{v}\right)_{w}$ have pure codimension 1 in $X_{w}^{v}$. Further, $X_{w}^{v}$ is normal and Cohen-Macaulay.

(2) The T-fixed points in $X_{w}^{v}$ are the $e_{x}$ where $x \in W^{P}$ and $v \leq x \leq w$.

(3) For $x, y$ in $W^{P}$, we have $X_{y}^{x} \subseteq X_{w}^{v} \Longleftrightarrow v \leq x \leq y \leq w$.

Proof. (2) is evident; it implies (3) and the first assertion of (1). To prove the remaining assertions, we use a variant of the argument of [1, Lemma 2. Consider the fiber product $G \times{ }^{B} X_{w}$ with projection map

$$
p: G \times{ }^{B} X_{w} \longrightarrow G / B,
$$

a $G$-equivariant locally trivial fibration with fiber $X_{w}$. We also have the "multiplication" map

$$
m: G \times^{B} X_{w} \longrightarrow G / P,(g, x) \longmapsto g x .
$$

This is a $G$-equivariant map to $G / P$; thus, it is also a locally trivial fibration. Its fiber $m^{-1}\left(e_{1}\right)$ is isomorphic to $\overline{P w^{-1} B} / B$ (a Schubert variety in $G / B$ ).

Next let $i: X^{v} \longrightarrow G / P$ be the inclusion and consider the cartesian product

$$
Z=X^{v} \times_{G / P}\left(G \times{ }^{B} X_{w}\right)
$$

with projections $\iota$ to $G \times{ }^{B} X_{w}, \mu$ to $X^{v}$ and $\pi$ to $G / B$, as displayed in the following commutative diagram:

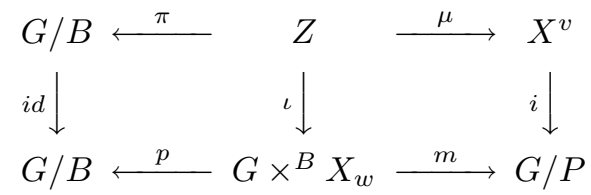

By definition, the square on the right is cartesian, so that $\mu$ is also a locally trivial fibration with fiber $\overline{P w^{-1} B} / B$ and base $X^{v}$. Since Schubert varieties are irreducible, normal and Cohen-Macaulay, it follows that the same holds for $Z$. Further, we have

$$
\operatorname{dim}(Z)=\operatorname{dim}\left(G \times{ }^{B} X_{w}\right)+\operatorname{dim}\left(X^{v}\right)-\operatorname{dim}(G / P)=\operatorname{dim}(G / B)+\ell(w)-\ell(v) .
$$

Notice that the fiber of $\pi: Z \longrightarrow G / B$ at each $g B / B$ identifies to the intersection $X^{v} \cap g X_{w}$; in particular, $\pi^{-1}(B / B)=X_{w}^{v}$. Notice also that $\iota: Z \longrightarrow G \times{ }^{B} X_{w}$ is a closed immersion with $B^{-}$-stable image (since this holds for $i: X^{v} \longrightarrow G / P$ ). Thus, $B^{-}$acts on $Z$ so that $\pi$ is equivariant. Since $B^{-} B / B$ is an open neighborhood of $B / B$ in $G / B$, isomorphic to $U^{-}$, its pullback under $\pi$ is an open subset of $Z$, isomorphic to $U^{-} \times X_{w}^{v}$. Therefore, $X_{w}^{v}$ is irreducible, normal and Cohen-Macaulay of dimension $\ell(w)-\ell(v)$.

We also record the following easy result, to be used in Section 7.

Lemma 2. Let $v \leq w$ in $W^{P}$, let $\lambda$ be a dominant character of $P$ and let $x(\lambda) \in$ $W^{\lambda}$. Then the restriction of $p_{x(\lambda)}$ to $X_{w}^{v}$ is nonzero if and only if $x(\lambda)$ admits a lift $x \in W^{P}$ such that $v \leq x \leq w$. Further, the ring

$$
\bigoplus_{n=0}^{\infty} H^{0}\left(X_{w}^{v}, L_{n \lambda}\right)
$$

is integral over its subring generated by the $\left.p_{x(\lambda)}\right|_{X_{w}^{v}}$ where $x \in W^{P}$ and $v \leq x \leq w$. 
Proof. Consider the natural map $G / P \rightarrow G / P_{\lambda}$ and its restriction $f: X_{w}^{v} \longrightarrow$ $f\left(X_{w}^{v}\right)$. The open subset $\left(p_{x(\lambda)} \neq 0\right)$ of $G / P_{\lambda}$ is affine, $T$-stable and contains $e_{x(\lambda)}$ as its unique closed $T$-orbit. Thus, $\left.p_{x(\lambda)}\right|_{X_{w}^{v}} \neq 0$ if and only if $e_{x(\lambda)} \in f\left(X_{w}^{v}\right)$. By Borel's fixed point theorem, this amounts to the existence of a $T$-fixed point $e_{x} \in X_{w}^{v}$ such that $f\left(e_{x}\right)=e_{x(\lambda)}$. Now Lemma $\square(2)$ completes the proof of the first assertion.

By the preceding arguments, the sections $\left.p_{x(\lambda)}\right|_{X_{w}^{v}}, x \in W^{P}, v \leq x \leq w$ do not vanish simultaneously at a $T$-fixed point of $X_{w}^{v}$. Since these sections are eigenvectors of $T$, it follows that they have no common zeroes. This implies the second assertion.

Remark. The image of a Richardson variety $X_{w}^{v}$ under a morphism $G / P \longrightarrow G / P_{\lambda}$ need not be another Richardson variety. Consider for example $G=S L(3)$ with simple reflections $s_{1}, s_{2}$. Let $P=B, w=s_{2} s_{1}, v=s_{2}$ and $\lambda=\omega_{1}$ (the fundamental weight fixed by $\left.s_{2}\right)$. Then $X_{w}^{v}$ is one-dimensional and mapped isomorphically to its image $f\left(X_{w}^{v}\right)$ in $G / P_{\lambda}$. Since the $T$-fixed points in $f\left(X_{w}^{v}\right)$ are $e_{\omega_{1}}$ and $e_{s_{2} s_{1}\left(\omega_{1}\right)}$, it follows that $f\left(X_{w}^{v}\right)$ is not a Richardson variety.

\section{COHOMOlOgy VANishing For RICHARDSON VARIETIES}

In this section, we assume that the characteristic of $k$ is $p>0$. Let $X$ be a scheme of finite type over $k$. Let $F: X \longrightarrow X$ be the absolute Frobenius morphism, that is, $F$ is the identity map on the topological space of $X$, and $F^{\#}: \mathcal{O}_{X} \longrightarrow F_{*} \mathcal{O}_{X}$ is the $p$-th power map. Then $X$ is called Frobenius split if the map $F^{\#}$ is split. We shall need a slight generalization of this notion, involving the composition $F^{r}=F \circ \cdots \circ F$ ( $r$ times), where $r$ is any positive integer.

Definition 2. We say that $X$ is split if there exists a positive integer $r$ such that the map

$$
\left(F^{r}\right)^{\#}: \mathcal{O}_{X} \longrightarrow F_{*}^{r} \mathcal{O}_{X}
$$

splits, that is, there exists an $\mathcal{O}_{X}$-linear map

$$
\varphi: F_{*}^{r} \mathcal{O}_{X} \longrightarrow \mathcal{O}_{X}
$$

such that $\varphi \circ\left(F^{r}\right)^{\#}$ is the identity; then $\varphi$ is called a splitting.

We shall also need a slight generalization of the notion of Frobenius splitting relative to an effective Cartier divisor (see [16]).

Definition 3. Let $X$ be a normal variety and $D$ an effective Weil divisor on $X$, with canonical section $s$. We say that $X$ is $D$-split if there exist a positive integer $r$ and an $\mathcal{O}_{X}$-linear map

$$
\psi: F_{*}^{r} \mathcal{O}_{X}(D) \longrightarrow \mathcal{O}_{X}
$$

such that the map

$$
\varphi: F_{*}^{r} \mathcal{O}_{X} \longrightarrow \mathcal{O}_{X}, f \longmapsto \psi(f s)
$$

is a splitting. Then $\psi$ is called a $D$-splitting.

We say that a closed subscheme $Y$ of $X$, with ideal sheaf $\mathcal{I}_{Y}$, is compatibly $D$-split if (a) no irreducible component of $Y$ is contained in the support of $D$, and (b) $\varphi\left(F_{*}^{r} \mathcal{I}_{Y}\right)=\mathcal{I}_{Y}$. 
Remarks. (i) Let $U$ be an open subset of $X$ such that $X-U$ has codimension at least 2 in $X$. Then $X$ is $D$-split if and only if $U$ is $D \cap U$-split (to see this, let $i: U \longrightarrow X$ be the inclusion, then $i_{*} \mathcal{O}_{U}=\mathcal{O}_{X}$ and $i_{*} \mathcal{O}_{U}(D \cap U)=\mathcal{O}_{X}(D)$ by normality of $X$ ).

Let $Y$ be a closed subscheme of $X$ such that $Y \cap U$ is dense in $Y$. Then $Y$ is compatibly $D$-split if and only if $Y \cap U$ is compatibly $D \cap U$-split (this is checked by the arguments of [16], 1.4-1.7).

(ii) If $X$ is split compatibly with an effective Weil divisor $D$, then $X$ is $\left(p^{r}-1\right) D$ split; to see this, one may assume that $X$ is nonsingular, by (i). Let $\varphi$ be a compatible splitting, then $\varphi\left(F_{*}^{r} \mathcal{O}_{X}(-D)\right)=\mathcal{O}_{X}(-D)$. Define $\psi: F_{*}^{r} \mathcal{O}_{X}(D) \longrightarrow$ $\mathcal{O}_{X}$ by $\psi\left(f \sigma^{p^{r}-1}\right)=\sigma \varphi\left(f \sigma^{-1}\right)$ for any local sections $f$ of $\mathcal{O}_{X}$ and $\sigma$ of $\mathcal{O}_{X}(D)$. Then one checks that $\psi$ is well defined, $\mathcal{O}_{X}$-linear and satisfies $\psi\left(f s^{p^{r}-1}\right)=\varphi(f)$.

(iii) Let $D$ and $E$ be effective Weil divisors in $X$, such that $D-E$ is effective. If $X$ is $D$-split, then it is $E$-split as well; if, in addition, a closed subscheme $Y$ of $X$ is compatibly $D$-split, then it is compatibly $E$-split (this follows from (i) together with [16], Remark 1.3 (ii)).

Lemma 3. Let $D, E$ be effective Weil divisors on a normal variety $X$, such that the support of $D$ contains the support of $E$. If $X$ is $D$-split, then $X$ is $E$-split as well. If, moreover, a closed subscheme $Y$ of $X$ is compatibly D-split, then $X$ is compatibly E-split.

Proof. Let $U$ be the set of those points of $X$ at which $D$ is a Cartier divisor. Then $U$ is an open subset with complement of codimension at least 2 (since $U$ contains the nonsingular locus of $X$ ). Moreover, $Y \cap U$ is dense in $Y$ (since $U$ contains the complement of the support of $D$ ). Thus, by Remark (i), we may replace $X$ with $U$, and hence assume that $D$ is a Cartier divisor.

Now let $\psi: F_{*}^{r} \mathcal{O}_{X}(D) \longrightarrow \mathcal{O}_{X}$ be a $D$-splitting. We regard $\psi$ as an additive map $\mathcal{O}_{X}(D) \longrightarrow \mathcal{O}_{X}$ such that $\psi(s)=1$, and $\psi\left(f^{p^{r}} \sigma\right)=f \psi(\sigma)$ for any local sections $f$ of $\mathcal{O}_{X}$ and $\sigma$ of $\mathcal{O}_{X}(D)$. For any positive integer $n$, we set

$$
\mathbf{n}=p^{r(n-1)}+p^{r(n-2)}+\cdots+1
$$

(then $\mathbf{1}=1$ ), and we define inductively a map

$$
\psi^{n}: F^{r n \#} \mathcal{O}_{X}(\mathbf{n} D) \longrightarrow \mathcal{O}_{X}
$$

by $\psi^{1}=\psi$, and

$$
\psi^{n}\left(f \sigma^{\mathbf{n}}\right)=\psi\left(\psi^{n-1}\left(f \sigma^{\mathbf{n}-1}\right) \sigma\right)
$$

for any local sections $f$ of $\mathcal{O}_{X}$ and $\sigma$ of $\mathcal{O}_{X}(D)$. Then one may check that $\psi^{n}$ is well defined and is a $\mathbf{n} D$-splitting of $X$. If, moreover, a closed subscheme $Y$ is compatibly $D$-split, then $\psi\left(F_{*}^{r}\left(\mathcal{I}_{Y} s\right)\right)=\mathcal{I}_{Y}$. By induction, it follows that $\psi^{n}\left(F_{*}^{r n}\left(\mathcal{I}_{Y} s^{\mathbf{n}}\right)\right)=\mathcal{I}_{Y}$, so that $Y$ is compatibly $\mathbf{n} D$-split.

Since the support of $D$ contains the support of $E$, there exists a positive integer $n$ such that $\mathbf{n} D-E$ is effective. Then $X$ is $\mathbf{n} D$-split, so that it is $E$-split by Remark (ii).

Lemma 4. Let $X$ be a normal projective variety endowed with an effective Weil divisor $D$ and with a globally generated line bundle $L$; let $Y$ be a closed subscheme of $X$. Assume that (a) $X$ is D-split compatibly with $Y$, and (b) the support of $D$ contains the support of an effective ample divisor. Then $H^{i}(X, L)=0=H^{i}(Y, L)$ for all $i \geq 1$, and the restriction map $H^{0}(X, L) \longrightarrow H^{0}(Y, L)$ is surjective. 
Proof. Choose an effective ample Cartier divisor $E$, with support contained in the support of $D$. Then $X$ is $E$-split compatibly with $Y$ by Lemma 3. Now the assertions follow from [16], 1.12 and 1.13.

We now apply this to Richardson varieties. By [16], 3.5, the variety $G / P$ is split compatibly with all Schubert varieties and with all opposite Schubert varieties; as a consequence, $G / P$ is split compatibly with all unions of Richardson varieties. By [16] 1.10, it follows that all scheme-theoretical intersections of unions of Richardson varieties are reduced; and using [16], 1.13, this also implies

Lemma 5. Let $\lambda$ be a regular dominant character of $P$ and let $Z$ be a union of Richardson varieties in $G / P$. Then the restriction map $H^{0}\left(G / P, L_{\lambda}\right) \rightarrow H^{0}\left(Z, L_{\lambda}\right)$ is surjective, and $H^{i}\left(Z, L_{\lambda}\right)=0$ for all $i \geq 1$. As a consequence, $H^{i}\left(X, L_{\lambda} \otimes \mathcal{I}_{Z}\right)=0$ for all $i \geq 1$.

Remark. If we only assume that $\lambda$ is dominant, then Lemma 5 extends to all unions of Schubert varieties (by [16]), but not to all unions of Richardson varieties. As a trivial example, take $G / P=\mathbb{P}^{1}$, the projective line with $T$-fixed points 0 and $\infty$, and $\lambda=0$. Then $Z:=\{0, \infty\}$ is a union of Richardson varieties, and the restriction map $H^{0}\left(\mathbb{P}^{1}, \mathcal{O}_{\mathbb{P}^{1}}\right) \longrightarrow H^{0}\left(Z, \mathcal{O}_{Z}\right)$ is not surjective. As a less trivial example, take $G / P=\mathbb{P}^{1} \times \mathbb{P}^{1}, Z=\left(\mathbb{P}^{1} \times\{0, \infty\}\right) \cup\left(\{0, \infty\} \times \mathbb{P}^{1}\right)$, and $\lambda=0$. Then $Z$ is again a union of Richardson varieties, and one checks that $H^{1}\left(Z, \mathcal{O}_{Z}\right) \neq 0$.

However, Lemma [5 does extend to all dominant characters and to unions of Richardson varieties with a common index.

Proposition 1. Let $\lambda$ be a dominant character of $P$ and let $Z$ be a union of Richardson varieties $X_{w}^{v}$ in $G / P$, all having the same $w$. Then the restriction $H^{0}\left(G / P, L_{\lambda}\right) \rightarrow H^{0}\left(Z, L_{\lambda}\right)$ is surjective, and $H^{i}\left(Z, L_{\lambda}\right)=0$ for all $i \geq 1$.

As a consequence, we have $H^{i}\left(X_{w}^{v}, L_{\lambda}(-Z)\right)=0$ for all $i \geq 1$, where $v \leq w$ in $W^{P}$, and $Z$ is a union of irreducible components of $\left(\partial X^{v}\right)_{w}$.

Proof. The Schubert variety $X_{w}$ is split compatibly with the effective Weil divisor $\partial X_{w}$ and with $Z$. By assumption, $\partial X_{w}$ contains no irreducible component of $Z$. Using Remarks (i) and (ii), it follows that $X_{w}$ is $(p-1) \partial X_{w}$-split compatibly with $Z$. Further, $\partial X_{w}$ is the support of an ample effective divisor, as follows from Chevalley's formula. Thus, Lemma 4 applies and yields surjectivity of $H^{0}\left(X_{w}, L_{\lambda}\right) \longrightarrow H^{0}\left(Z, L_{\lambda}\right)$ together with vanishing of $H^{i}\left(Z, L_{\lambda}\right)$ for $i \geq 1$. Now surjectivity of $H^{0}\left(G / P, L_{\lambda}\right) \longrightarrow H^{0}\left(X_{w}, L_{\lambda}\right)$ completes the proof of the first assertion.

In particular, we have $H^{i}\left(X_{w}^{v}, L_{\lambda}\right)=H^{i}\left(Z, L_{\lambda}\right)=0$ for all $i \geq 1$, and the restriction map $H^{0}\left(X_{w}^{v}, L_{\lambda}\right) \longrightarrow H^{0}\left(Z, L_{\lambda}\right)$ is surjective; this implies the second assertion.

We shall also need the following, more technical vanishing result.

Proposition 2. Let $\lambda$ be a dominant character of $P$ and let $v, w$ in $W^{P}$ such that $v \leq w$. Then

$$
H^{i}\left(X_{w}^{v}, L_{\lambda}\left(-\left(\partial_{\lambda} X_{w}\right)^{v}-Z\right)\right)=0
$$

for any $i \geq 1$ and for any (possibly empty) union $Z$ of irreducible components of $\left(\partial X^{v}\right)_{w}$. 
Proof. We shall rely on the following result (see [13] Theorem 1). Let $\pi: X \longrightarrow Y$ be a proper morphism of schemes. Let $D$ (resp. E) be a closed subscheme of $X$ (resp. $Y$ ) and let $i$ be a positive integer such that:

(i) $\pi^{-1}(E)$ is contained in $D$ (as sets).

(ii) $R^{i} \pi_{*}\left(\mathcal{I}_{D}\right)=0$ outside $E$.

(iii) $X$ is split compatibly with $D$.

Then $R^{i} \pi_{*}\left(\mathcal{I}_{D}\right)=0$ everywhere.

To apply this result, consider the restriction

$$
f: X_{w}^{v} \longrightarrow f\left(X_{w}^{v}\right)
$$

of the natural map $G / P \longrightarrow G / P_{\lambda}$. Then $L_{\lambda}=f^{*} M_{\lambda}$ for a very ample line bundle $M_{\lambda}$ on $f\left(X_{w}^{v}\right)$. Let $Y$ be the corresponding affine cone over $f\left(X_{w}^{v}\right)$, with vertex 0 and projection map

$$
q: Y-\{0\} \longrightarrow f\left(X_{w}^{v}\right) .
$$

And let $X$ be the total space of the line bundle $L_{-\lambda}$ (dual to $L_{\lambda}$ ), with projection map

$$
p: X \longrightarrow X_{w}^{v}
$$

and zero section $X_{0}$. Then the algebra

$$
H^{0}\left(X, \mathcal{O}_{X}\right)=\bigoplus_{n=0}^{\infty} H^{0}\left(X_{w}^{v}, L_{n \lambda}\right)
$$

contains $H^{0}\left(Y, \mathcal{O}_{Y}\right)$ as the subalgebra generated by $H^{0}\left(f\left(X_{w}^{v}\right), M_{\lambda}\right)$. The algebra $H^{0}\left(X, \mathcal{O}_{X}\right)$ is finitely generated, and the corresponding morphism

$$
X \longrightarrow \operatorname{Spec} H^{0}\left(X, \mathcal{O}_{X}\right)
$$

is proper, since the line bundle $L_{\lambda}$ is globally generated. Moreover, since $L_{\lambda}$ is the pullback under $f$ of the very ample line bundle $M_{\lambda}$, the algebra $H^{0}\left(X, \mathcal{O}_{X}\right)$ is a finite module over its subalgebra $H^{0}\left(Y, \mathcal{O}_{Y}\right)$. This defines a proper morphism $\pi: X \longrightarrow Y$, and we have $\pi^{-1}(0)=X_{0}$ (as sets). Moreover, the diagram

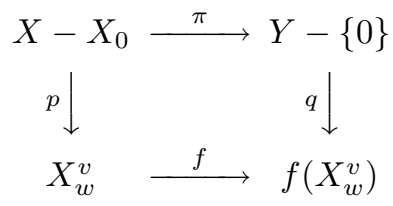

is cartesian, and the vertical maps are principal $\mathbb{G}_{m}$-bundles.

Now let $D=X_{0} \cup p^{-1}\left(\left(\partial_{\lambda} X_{w}\right)^{v} \cup Z\right)$; this is a closed subscheme of $X$ with ideal sheaf

$$
\mathcal{I}_{D}=p^{*} L_{\lambda}\left(-\left(\partial_{\lambda} X_{w}\right)^{v}-Z\right) .
$$

Let $E$ be the affine cone over $f\left(\left(\partial_{\lambda} X_{w}\right)^{v}\right)$; this is a closed subscheme of $Y$. We check that the conditions (i), (ii) and (iii) hold.

For (i), notice that

$$
\pi^{-1}(E)=X_{0} \cup p^{-1} f^{-1} f\left(\left(\partial_{\lambda} X_{w}\right)^{v}\right)=X_{0} \cup p^{-1}\left(\left(\partial_{\lambda} X_{w}\right)^{v}\right)
$$

(as sets), by the definition of $\left(\partial_{\lambda} X_{w}\right)^{v}$. In other words, $\pi^{-1}(E) \subseteq D$ as sets.

For (ii), observe that $\mathcal{I}_{D}=p^{*} L_{\lambda}(-Z)$ outside $\pi^{-1}(E)$. Thus, (ii) is equivalent to $R^{i} \pi_{*}\left(p^{*} L_{\lambda}(-Z)\right)=0$ outside $E$. We show that $R^{i} \pi_{*}\left(p^{*} L_{\lambda}(-Z)\right)=0$ outside 0 . Using the cartesian square above, it suffices to check that $R^{i} f_{*}\left(L_{\lambda}(-Z)\right)=0$; 
by the Leray spectral sequence and the Serre vanishing theorem, this amounts to $H^{i}\left(X_{w}^{v}, L_{n \lambda}(-Z)\right)=0$ for large $n$. But this holds by Proposition 1 .

For (iii), recall that $X_{w}^{v}$ is split compatibly with $\left(\partial_{\lambda} X_{w}\right)^{v} \cup Z$. Let $\varphi$ be a compatible splitting; then $\varphi$ lifts uniquely to a splitting of $X$ compatibly with $X_{0}$ and with $p^{-1}\left(\left(\partial_{\lambda} X_{w}\right)^{v} \cup Z\right)$. It follows that $X$ is split compatibly with $D$.

We thus obtain $R^{i} \pi_{*}\left(\mathcal{I}_{D}\right)=0$ for all $i \geq 1$. Since $Y$ is affine, this amounts to $H^{i}\left(X, \mathcal{I}_{D}\right)=0$ for all $i \geq 1$. On the other hand, since the morphism $p: X \longrightarrow X_{w}^{v}$ is affine, we obtain that $H^{i}\left(X, \mathcal{I}_{D}\right)$ is isomorphic to

$$
H^{i}\left(X_{w}^{v}, p_{*} p^{*}\left(L_{\lambda}\left(-\left(\partial_{\lambda} X_{w}\right)^{v}\right)-Z\right)\right)=H^{i}\left(X_{w}^{v}, L_{\lambda}\left(-\left(\partial_{\lambda} X_{w}\right)^{v}-Z\right) \otimes p_{*} \mathcal{O}_{X}\right) .
$$

Further, $p_{*} \mathcal{O}_{X}$ contains $\mathcal{O}_{X_{w}^{v}}$ as a direct factor. This yields

$$
H^{i}\left(X_{w}^{v}, L_{\lambda}\left(-\left(\partial_{\lambda} X_{w}\right)^{v}-Z\right)\right)=0
$$

for all $i \geq 1$.

Corollary 1. With the above notations, the restriction map

$$
H^{0}\left(X_{w}^{v}, L_{\lambda}\left(-\left(\partial_{\lambda} X_{w}\right)^{v}\right)\right) \longrightarrow H^{0}\left(X_{w}^{x}, L_{\lambda}\left(-\left(\partial_{\lambda} X_{w}\right)^{x}\right)\right)
$$

is surjective for any $x \in W^{P}$ such that $v \leq x \leq w$.

Proof. We may reduce to the case that $\ell(x)=\ell(v)+1$, that is, $X_{w}^{x}$ is an irreducible component of $\left(\partial X^{v}\right)_{w}$. Then $H^{1}\left(X_{w}^{v}, L_{\lambda}\left(-\left(\partial_{\lambda} X_{w}\right)^{v}-X_{w}^{x}\right)\right)=0$ by Proposition 2 Now the assertion follows from the exact sequence

$$
\left.\left.\left.0 \rightarrow L_{\lambda}\right|_{X_{w}^{v}}\left(-\left(\partial_{\lambda} X_{w}\right)^{v}-X_{w}^{x}\right) \rightarrow L_{\lambda}\right|_{X_{w}^{v}}\left(-\left(\partial_{\lambda} X_{w}\right)^{v}\right) \rightarrow L_{\lambda}\right|_{X_{w}^{x}}\left(-\left(\partial_{\lambda} X_{w}\right)^{x}\right) \rightarrow 0 .
$$

Notice finally that Lemma 5 Propositions 1 and 2 and Corollary 1 also hold in characteristic zero, as follows from the argument in [16], 3.7.

\section{Filtrations}

In this section, we shall obtain natural filtrations of the $T$-modules $H^{0}\left(X_{w}^{v}, L_{\lambda}\right)$ and $H^{0}\left(X_{w}^{v}, L_{\lambda}\left(-\left(\partial_{\lambda} X_{w}\right)^{v}\right)\right.$ ) (where $X_{w}^{v}$ is a Richardson variety in $G / P$, and $\lambda$ is a dominant character of $P$ ), and we shall describe their associated graded modules. For this, we shall construct a degeneration of $X_{w}^{v}$ embedded diagonally in $G / P \times$ $G / P$, to a union of products of Richardson varieties.

Such a degeneration was obtained in [2, Theorem 16 for $X_{w}^{v}=G / B$, by using the wonderful compactification of the adjoint group of $G$; it was extended to certain subvarieties in $G / P$, including Schubert varieties, in [1, Theorem 2. Here we follow a direct, self-contained approach, at the cost of repeating some of the arguments in 2] and [1. We begin by establishing a Künneth decomposition of the class of the diagonal of $G / P$, in the Grothendieck group of $G / P \times G / P$; such a decomposition is deduced in [2] from a degeneration of the diagonal.

Let $K(G / P \times G / P)$ be the Grothendieck group of the category of coherent sheaves on $G / P \times G / P$. The class of a coherent sheaf $\mathcal{F}$ in this group will be denoted by $[\mathcal{F}]$.

Lemma 6. We have in $K(G / P \times G / P)$ :

$$
\begin{array}{r}
{\left[\mathcal{O}_{\operatorname{diag}(G / P)}\right]=\left[\mathcal{O}_{\cup_{x \in W^{P}}} X_{x} \times X^{x}\right]} \\
=\sum_{x \in W^{P}}\left[\mathcal{O}_{X_{x}}\left(-\partial X_{x}\right) \otimes \mathcal{O}_{X^{x}}\right]=\sum_{x \in W^{P}}\left[\mathcal{O}_{X_{x}} \otimes \mathcal{O}_{X^{x}}\left(-\partial X^{x}\right)\right] .
\end{array}
$$


Proof. Let $Z=\bigcup_{x \in W^{P}} X_{x} \times X^{x}$. We first claim that

$$
\left[\mathcal{O}_{Z}\right]=\sum_{x \in W^{P}}\left[\mathcal{O}_{X_{x}}\left(-\partial X_{x}\right) \otimes \mathcal{O}_{X^{x}}\right] .
$$

Let $W^{P}=\left\{x_{1}, \ldots, x_{N}\right\}$ be an indexing such that $i \leq j$ whenever $x_{i} \leq x_{j}$. Then one obtains easily

$$
\left(X_{x_{i}} \times X^{x_{i}}\right) \cap\left(\bigcup_{j<i} X_{x_{j}} \times X^{x_{j}}\right)=\partial X_{x_{i}} \times X^{x_{i}}
$$

Now let $\mathcal{O}_{Z, \geq i}$ be the subsheaf of $\mathcal{O}_{Z}$ consisting of those sections that vanish on $X_{x_{j}} \times X^{x_{j}}$ for each $j<i$. Then the $\mathcal{O}_{Z, \geq i}$ are a decreasing filtration of $\mathcal{O}_{Z}$, and

$$
\mathcal{O}_{Z, \geq i} /\left.\mathcal{O}_{Z, \geq i+1} \simeq \mathcal{O}_{Z, \geq i}\right|_{X_{x_{i}} \times X^{x_{i}}} \simeq \mathcal{O}_{X_{i}}\left(-\partial X_{i}\right) \otimes \mathcal{O}_{X^{i}} .
$$

Further, $\left[\mathcal{O}_{Z}\right]=\sum_{i=1}^{N}\left[\mathcal{O}_{Z, \geq i} / \mathcal{O}_{Z, \geq i+1}\right]$ in $K(G / P \times G / P)$. This implies our claim.

One checks similarly that

$$
\left[\mathcal{O}_{Z}\right]=\sum_{x \in W^{P}}\left[\mathcal{O}_{X_{x}} \otimes \mathcal{O}_{X^{x}}\left(-\partial X^{x}\right)\right]
$$

using the increasing filtration of $\mathcal{O}_{Z}$ by the subsheaves $\mathcal{O}_{Z, \leq i}$ consisting of those sections that vanish on $X_{x_{j}} \times X^{x_{j}}$ for each $j>i$.

To complete the proof, it suffices to check that

$$
\left[\mathcal{O}_{\operatorname{diag}(G / P)}\right]=\sum_{x \in W^{P}}\left[\mathcal{O}_{X_{x}} \otimes \mathcal{O}_{X^{x}}\left(-\partial X^{x}\right)\right]
$$

For this, we recall some well-known facts on Grothendieck groups of flag varieties.

Since the Bruhat cells $C_{x}, x \in W^{P}$, form a cellular decomposition of $G / P$, the abelian group $K(G / P)$ is generated by the $\left[\mathcal{O}_{X_{x}}\right], x \in W^{P}$. Likewise, it is generated by the $\left[\mathcal{O}_{X^{y}}\right], y \in W^{P}$. Further, $K(G / P)$ is a ring for the product

$$
[\mathcal{F}] \cdot[\mathcal{G}]=\sum_{i \geq 0}(-1)^{i}\left[\operatorname{Tor}_{i}^{G / P}(\mathcal{F}, \mathcal{G})\right]
$$

and the Euler characteristic of coherent sheaves yields an additive map

$$
\begin{aligned}
\chi: K(G / P) & \longrightarrow \mathbb{Z} \\
{[\mathcal{F}] } & \longmapsto \chi(\mathcal{F}) .
\end{aligned}
$$

Since $X_{x}$ and $X^{y}$ are Cohen-Macaulay and intersect properly in $G / P$, we have $\operatorname{Tor}_{i}^{G / P}\left(\mathcal{O}_{X_{x}}, \mathcal{O}_{X^{y}}\right)=0$ for all $i \geq 1$ (see [1], Lemma 1 for details). And since the intersection $X_{x} \cap X^{y}=X_{x}^{y}$ is reduced, we obtain

$$
\operatorname{Tor}_{0}^{G / P}\left(\mathcal{O}_{X_{x}}, \mathcal{O}_{X^{y}}\right)=\mathcal{O}_{X_{x}} \otimes_{\mathcal{O}_{G / P}} \mathcal{O}_{X^{y}}= \begin{cases}\mathcal{O}_{X_{x}^{y}} & \text { if } y \leq x, \\ 0 & \text { otherwise. }\end{cases}
$$

Together with Proposition [1, it follows that

$$
\chi\left(\left[\mathcal{O}_{X_{x}}\right] \cdot\left[\mathcal{O}_{X^{y}}\right]\right)= \begin{cases}1 & \text { if } y \leq x, \\ 0 & \text { otherwise. }\end{cases}
$$

On the other hand, we have in $K(G / P)$,

$$
\left[\mathcal{O}_{X^{y}}\right]=\sum_{z \in W^{P}, z \geq y}\left[\mathcal{O}_{X^{z}}\left(-\partial X^{z}\right)\right]
$$


(more generally, for any union $Z$ of opposite Schubert varieties, we have $\left[\mathcal{O}_{Z}\right]=$ $\sum_{z \in W^{P}, X^{z} \subseteq Z}\left[\mathcal{O}_{X^{z}}\left(-\partial X^{z}\right)\right]$ by an easy induction, using the fact that intersections of unions of opposite Schubert varieties are reduced.) It follows that

$$
\chi\left(\left[\mathcal{O}_{X_{x}}\right] \cdot\left[\mathcal{O}_{X^{y}}\left(-\partial X^{y}\right)\right]\right)=\delta_{x, y} .
$$

Thus, the $\left[\mathcal{O}_{X_{x}}\right], x \in W^{P}$ form a basis for $K(G / P)$; further, the bilinear form $K(G / P) \times K(G / P) \longrightarrow \mathbb{Z},(u, v) \longmapsto \chi(u \cdot v)$ is nondegenerate, and the dual basis of the $\left[\mathcal{O}_{X_{x}}\right]$ with respect to this pairing consists of the $\left[\mathcal{O}_{X^{x}}\left(-\partial X^{x}\right)\right]$.

It follows that a given class $u \in K(G / P \times G / P)$ is zero if and only if $\chi\left(u \cdot\left[\mathcal{O}_{X^{y}}\left(-\partial X^{y}\right) \otimes \mathcal{O}_{X_{z}}\right]\right)=0$ for all $y, z \in W^{P}$. Further,

$$
\chi\left(\left[\mathcal{O}_{\operatorname{diag}(G / P)}\right] \cdot\left[\mathcal{O}_{X^{y}}\left(-\partial X^{y}\right) \otimes \mathcal{O}_{X_{z}}\right]\right)=\chi\left(\left[\mathcal{O}_{X^{y}}\left(-\partial X^{y}\right)\right] \cdot\left[\mathcal{O}_{X_{z}}\right]\right)=\delta_{y, z} ;
$$

whereas

$$
\begin{aligned}
& \chi\left(\sum_{x \in W^{P}}\left[\mathcal{O}_{X_{x}} \otimes \mathcal{O}_{X^{x}}\left(-\partial X^{x}\right)\right] \cdot\left[\mathcal{O}_{X^{y}}\left(-\partial X^{y}\right) \otimes \mathcal{O}_{X_{z}}\right]\right) \\
& =\sum_{x \in W^{P}} \chi\left(\left[\mathcal{O}_{X_{x}}\right] \cdot\left[\mathcal{O}_{X^{y}}\left(-\partial X^{y}\right)\right]\right) \chi\left(\left[\mathcal{O}_{X^{x}}\left(-\partial X^{x}\right)\right] \cdot\left[\mathcal{O}_{X_{z}}\right]\right)=\sum_{x \in W^{P}} \delta_{x, y} \delta_{x, z}=\delta_{y, z} .
\end{aligned}
$$

This completes the proof of $(*)$, and hence of the lemma.

We now construct a degeneration of the diagonal of any Richardson variety. Let $\theta: \mathbb{G}_{m} \longrightarrow T$ be a regular dominant one-parameter subgroup. Let $\mathcal{X}$ be the closure in $G / P \times G / P \times \mathbb{A}^{1}$ of the subset

$$
\left\{(x, \theta(s) x, s) \mid x \in G / P, s \in k^{*}\right\} .
$$

The variety $\mathcal{X}$ is invariant under the action of $\mathbb{G}_{m} \times T$ defined by

$$
(s, t)(x, y, z)=(t x, \theta(s) t y, s z) .
$$

Consider the projections

$$
p_{1}, p_{2}: \mathcal{X} \longrightarrow G / P, \pi: \mathcal{X} \longrightarrow \mathbb{A}^{1} .
$$

Clearly, $\pi$ is proper and flat, and its fibers identify with closed subschemes of $G / P \times G / P$ via $p_{1} \times p_{2}$; this identifies the fiber at 1 with $\operatorname{diag}(G / P) \simeq G / P$. By equivariance, every "general" fiber $\pi^{-1}(z)$, where $z \neq 0$, is also isomorphic to $G / P$.

We shall denote the "special" (scheme-theoretical) fiber $\pi^{-1}(0)$ by $F$, with projections

$$
q_{1}, q_{2}: F \longrightarrow G / P \text {. }
$$

Next let $v, w$ in $W^{P}$ such that $v \leq w$. Let $\mathcal{X}_{w}^{v}$ be the closure in $G / P \times G / P \times \mathbb{A}^{1}$ of the subset

$$
\left\{(x, \theta(s) x, s) \mid x \in X_{w}^{v}, s \in k^{*}\right\} .
$$

This is a subvariety of $\mathcal{X} \cap\left(X_{w}^{v} \times X_{w}^{v} \times \mathbb{A}^{1}\right)$, invariant under the action of $\mathbb{G}_{m} \times T$. We shall denote the restrictions of $p_{1}, p_{2}, \pi$ to $\mathcal{X}_{w}^{v}$ by the same letters; then $\pi$ is again proper and flat, and its "general" fibers are isomorphic to $X_{w}^{v}$. Let $F_{w}^{v}$ be the "special" fiber, with projections $q_{1}, q_{2}$ to $X_{w}^{v}$.

Lemma 7. (1) The schemes $F$ and $F_{w}^{v}$ are reduced. Further,

$$
F=\bigcup_{x \in W^{P}} X_{x} \times X^{x} \text { and } F_{w}^{v}=\bigcup_{x \in W^{P}, v \leq x \leq w} X_{x}^{v} \times X_{w}^{x} \text {. }
$$


(2) Choose a total ordering $\leq_{t}$ of $W^{P}$ such that $x \leq_{t} y$ whenever $x \leq y$. For $x \in W^{P}$, let $\mathcal{O}_{F, \leq_{t} x}$ (resp. $\mathcal{O}_{F, \geq_{t} x}$ ) be the subsheaf of $\mathcal{O}_{F}$ consisting of those sections that vanish identically on $X_{y} \times X^{y}$ for each $y>_{t} x$ (resp. $y<_{t}$ $x$ ). Then the $\mathcal{O}_{F, \leq_{t} x}$ (resp. $\mathcal{O}_{F, \geq_{t} x}$ ) are an ascending (resp. descending) filtration of $\mathcal{O}_{F}$, with associated graded

$$
\bigoplus_{x \in W^{P}} \mathcal{O}_{X_{x}} \otimes \mathcal{O}_{X^{x}}\left(-\partial X^{x}\right), \text { resp. } \bigoplus_{x \in W^{P}} \mathcal{O}_{X_{x}}\left(-\partial X_{x}\right) \otimes \mathcal{O}_{X^{x}}
$$

The induced filtrations on the structure sheaf $\mathcal{O}_{F_{w}^{v}}$ have associated graded

$$
\bigoplus_{x \in W^{P}, v \leq x \leq w} \mathcal{O}_{X_{x}^{v}} \otimes \mathcal{O}_{X_{w}^{x}}\left(-\left(\partial X^{x}\right)_{w}\right), \text { resp. } \bigoplus_{x \in W^{P}, v \leq x \leq w} \mathcal{O}_{X_{x}^{v}}\left(-\left(\partial X_{x}\right)^{v}\right) \otimes \mathcal{O}_{X_{w}^{x}} .
$$

The induced map

$$
\mathcal{O}_{F_{w}^{v}, \leq_{t}} \longrightarrow \mathrm{gr}_{\leq t} \mathcal{O}_{F_{w}^{v}}
$$

is just the restriction to $X_{x}^{v} \times X_{w}^{x}$; the same holds for the induced map

$$
\mathcal{O}_{F_{w}^{v}, \geq_{t}} \longrightarrow \operatorname{gr}_{\geq t} \mathcal{O}_{F_{w}^{v}}
$$

Proof. (1) Let $x \in W^{P}$. We claim that

$$
C_{x} \times C^{x} \subseteq F .
$$

To check this, consider the subset $x C^{1}$ of $G / P$. This is an open $T$-stable neighborhood of $e_{x}$ in $G / P$, isomorphic to affine space where $T$ acts linearly with weights the $\alpha \in x\left(R^{-}-R_{P}\right)$. Choose corresponding coordinate functions $z_{\alpha}$ on $x C^{1}$, then $C_{x}$ (resp. $C^{x}$ ) is the closed subset of $x C^{1}$ where $z_{\alpha}=0$ whenever $\alpha \in R^{-}$ (resp. $\alpha \in R^{+}$). Let $z=\left(z_{\alpha}\right) \in x C^{1}$, then

$$
\theta(s) z=\left(s^{\langle\alpha, \theta\rangle} z_{\alpha}\right) .
$$

Denote by $z_{+}$(resp. $z_{-}$) the point of $C_{x}$ (resp. $C^{x}$ ) with coordinates $z_{\alpha}, \alpha \in R^{+}$ (resp. $\alpha \in R^{-}$). Let $z^{\prime}(s)$ be the point of $x C^{1}$ with $\alpha$-coordinate $z_{\alpha}$ if $\alpha \in R^{+}$, and $\theta\left(s^{-1}\right) z_{\alpha}$ otherwise. Since $\theta$ is regular dominant, we obtain

$$
\lim _{s \rightarrow 0}\left(z^{\prime}(s), \theta(s) z^{\prime}(s), s\right)=\left(z_{+}, z_{-}, 0\right) .
$$

Since $z_{+}$(resp. $\left.z_{-}\right)$is an arbitrary point of $C_{x}$ (resp. $C^{x}$ ), this proves our claim.

The claim implies that $F$ contains $\bigcup_{x \in W^{P}} X_{x} \times X^{x}$ as a reduced closed subscheme. Let $\mathcal{I}$ be the ideal sheaf of this closed subscheme in $\mathcal{O}_{F}$; we regard $\mathcal{I}$ as a coherent sheaf on $G / P \times G / P$. Then we have in $K(G / P \times G / P)$ :

$$
[\mathcal{I}]=\left[\mathcal{O}_{F}\right]-\left[\mathcal{O}_{\bigcup_{x \in W^{P}} X_{x} \times X^{x}}\right]=\left[\mathcal{O}_{\operatorname{diag}(G / P}\right]-\left[\mathcal{O}_{\cup_{x \in W^{P}} X_{x} \times X^{x}}\right]=0,
$$

where the first equality follows from the definition of $\mathcal{I}$, the second one from the fact that $\pi: \mathcal{X} \rightarrow \mathbb{A}^{1}$ is flat with fibers $F$ and $\operatorname{diag}(G / P)$, and the third one from Lemma 6. As a consequence, $\mathcal{I}$ is trivial (e.g., since its Hilbert polynomial is zero); this completes the proof for $F$.

In the case of $F_{w}^{v}$, notice that

$$
F_{w}^{v} \subseteq F \cap\left(X^{v} \times X_{w}\right)=\left(\bigcup_{x \in W^{P}} X_{x} \times X^{x}\right) \cap\left(X^{v} \times X_{w}\right)=\bigcup_{x \in W^{P}, v \leq x \leq w} X_{x}^{v} \times X_{w}^{x}
$$


as schemes, since all involved scheme-theoretic intersections are reduced. Further, we have in the Chow ring of $G / P \times G / P$ :

$$
\begin{aligned}
{\left[F_{w}^{v}\right]=\left[\operatorname{diag}\left(X_{w}^{v}\right)\right] } & =\left[\operatorname{diag}(G / P) \cap\left(X^{v} \times X_{w}\right)\right]=[\operatorname{diag}(G / P)] \cdot\left[X^{v} \times X_{w}\right] \\
= & {[F] \cdot\left[X^{v} \times X_{w}\right]=\left[F \cap\left(X^{v} \times X_{w}\right)\right]=\sum_{x \in W^{P}, v \leq x \leq w}\left[X_{x}^{v} \times X_{w}^{x}\right], }
\end{aligned}
$$

since all involved intersections are proper and reduced. It follows that $F_{w}^{v}$ equals $\bigcup_{x \in W^{P}, v \leq x \leq w} X_{x}^{v} \times X_{w}^{x}$.

(2) has been established in the case of $F$, at the beginning of the proof of Lemma 6] The general case is similar.

Next let $\lambda$ be a dominant character of $P$. This yields $T$-linearized line bundles $q_{2}^{*} L_{\lambda}$ on $F$ and on $F_{w}^{v}$, together with "adjunction" maps $H^{0}\left(G / P, L_{\lambda}\right) \longrightarrow$ $H^{0}\left(F, q_{2}^{*} L_{\lambda}\right)$ and $H^{0}\left(X_{w}^{v}, L_{\lambda}\right) \longrightarrow H^{0}\left(F_{w}^{v}, q_{2}^{*} L_{\lambda}\right)$.

Proposition 3. (1) These maps are isomorphisms, and the restriction map

$$
H^{0}\left(F, q_{2}^{*} L_{\lambda}\right) \longrightarrow H^{0}\left(F_{w}^{v}, q_{2}^{*} L_{\lambda}\right)
$$

is surjective.

(2) The ascending filtration of $\mathcal{O}_{F}$ yields an ascending filtration of the $T$ module $H^{0}\left(F, q_{2}^{*} L_{\lambda}\right)$, with associated graded

$$
\bigoplus_{x \in W^{P}} H^{0}\left(X^{x}, L_{\lambda}\left(-\partial X^{x}\right)\right) .
$$

(3) The image of this filtration under restriction to $F_{w}^{v}$ has associated graded

$$
\bigoplus_{x \in W^{P}, v \leq x \leq w} H^{0}\left(X_{w}^{x}, L_{\lambda}\left(-\left(\partial X^{x}\right)_{w}\right)\right) .
$$

Hence this is the associated graded of an ascending filtration

$$
H^{0}\left(X_{w}^{v}, L_{\lambda}\right){ }_{t} x, \quad v \leq x \leq w
$$

of $H^{0}\left(X_{w}^{v}, L_{\lambda}\right)$, compatible with the $T$-action and restrictions to smaller Richardson varieties.

(4) The subspace

$$
H^{0}\left(X_{w}^{v}, L_{\lambda}\right)_{{ }_{t} x} \subseteq H^{0}\left(X_{w}^{v}, L_{\lambda}\right)
$$

consists of those sections that vanish identically on $X_{w}^{y}$ for all $y>_{t} x$. Further, the map

$$
H^{0}\left(X_{w}^{v}, L_{\lambda}\right)_{\leq_{t} x} \longrightarrow \operatorname{gr}_{x} H^{0}\left(X_{w}^{v}, L_{\lambda}\right)=H^{0}\left(X_{w}^{x}, L_{\lambda}\left(-\left(\partial X^{x}\right)_{w}\right)\right)
$$

is just the restriction to $X_{w}^{x}$.

Proof. (1) We have

$$
H^{0}\left(F, q_{2}^{*} L_{\lambda}\right)=H^{0}\left(G / P, q_{2 *} q_{2}^{*} L_{\lambda}\right)=H^{0}\left(G / P, L_{\lambda} \otimes q_{2 *} \mathcal{O}_{F}\right)
$$

by the projection formula. Further, the associated graded of the descending filtration of $\mathcal{O}_{F}$ is acyclic for $q_{2 *}$; indeed, $H^{i}\left(X_{x}, \mathcal{O}_{X_{x}}\left(-\partial X_{x}\right)\right)=0$ for all $i \geq 1$ and all $x \in W^{P}$, by Proposition 1 Notice also that $H^{0}\left(X_{x}, \mathcal{O}_{X_{x}}\left(-\partial X_{x}\right)\right)=\overline{0}$ for all $x \neq 1$, since $\partial X_{x}$ is a nonempty subscheme of the complete variety $X_{x}$. It follows that the natural map $\mathcal{O}_{G / P} \longrightarrow q_{2 *} \mathcal{O}_{F}$ is an isomorphism. Hence the same holds for the map $H^{0}\left(G / P, L_{\lambda}\right) \longrightarrow H^{0}\left(F, q_{2}^{*} L_{\lambda}\right)$. 
Likewise, the map $H^{0}\left(X_{w}^{v}, L_{\lambda}\right) \longrightarrow H^{0}\left(F_{w}^{v}, q_{2}^{*} L_{\lambda}\right)$ is an isomorphism as well. Since the restriction map $H^{0}\left(G / P, L_{\lambda}\right) \longrightarrow H^{0}\left(X_{w}^{v}, L_{\lambda}\right)$ is surjective by Proposition 10 the same holds for $H^{0}\left(F, q_{2}^{*} L_{\lambda}\right) \longrightarrow H^{0}\left(F_{w}^{v}, q_{2}^{*} L_{\lambda}\right)$.

(2) By Lemma 7 again, the ascending filtration of $\mathcal{O}_{F}$ yields one on $q_{2}^{*} L_{\lambda}$, with associated graded

$$
\left.\bigoplus_{x \in W^{P}} \mathcal{O}_{X_{x}} \otimes L_{\lambda}\right|_{X^{x}}\left(-\partial X^{x}\right)
$$

The latter is acyclic by Proposition 1 It follows that $H^{0}\left(F, q_{2}^{*} L_{\lambda}\right)$ has an ascending filtration with associated graded as claimed.

(3) is checked similarly.

(4) We have

$$
\begin{aligned}
& H^{0}\left(X_{w}^{v}, L_{\lambda}\right)_{\leq_{t} x}=H^{0}\left(F_{w}^{v}, q_{2}^{*} L_{\lambda}\right)_{\leq_{t} x}=H^{0}\left(F_{w}^{v}, q_{2}^{*} L_{\lambda} \otimes \mathcal{I}_{\cup_{y>t_{t} x} X_{y}^{v} \times X_{w}^{y}}\right) \\
& =H^{0}\left(X_{w}^{v}, L_{\lambda} \otimes q_{2 *} \mathcal{I}_{\cup_{y>t} X_{y}^{v} \times X_{w}^{y}}\right)
\end{aligned}
$$

by the projection formula. Further, $q_{2 *} \mathcal{O}_{F_{w}^{v}}=\mathcal{O}_{X_{w}^{v}}$ as seen in the proof of (1). It follows that

This implies our statement.

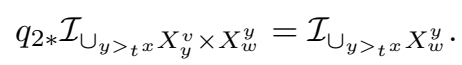

We now construct a similar filtration of the $T$-submodule

$$
H^{0}\left(X_{w}^{v}, L_{\lambda}\left(-\left(\partial_{\lambda} X_{w}\right)^{v}\right)\right) \subseteq H^{0}\left(X_{w}^{v}, L_{\lambda}\right) .
$$

For this, we define a sheaf on $F_{w}^{v}$ by

$$
q_{2}^{*} L_{\lambda}\left(-\left(\partial_{\lambda} X_{w}\right)^{v}\right)=\left(q_{2}^{*} L_{\lambda}\right) \otimes_{\mathcal{O}_{F_{w}^{v}}} \mathcal{I}_{q_{2}^{-1}\left(\left(\partial_{\lambda} X_{w}\right)^{v}\right)}
$$

This is a subsheaf of $q_{2}^{*} L_{\lambda}$; it may differ from the pullback sheaf of $L_{\lambda}\left(-\left(\partial_{\lambda} X_{w}\right)^{v}\right)$ under $q_{2}$. We also have an "adjunction" map

$$
H^{0}\left(X_{w}^{v}, L_{\lambda}\left(-\left(\partial_{\lambda} X_{w}\right)^{v}\right)\right) \longrightarrow H^{0}\left(F_{w}^{v}, q_{2}^{*} L_{\lambda}\left(-\left(\partial_{\lambda} X_{w}\right)^{v}\right)\right) .
$$

In particular, we obtain a map

$$
H^{0}\left(X_{w}, L_{\lambda}\left(-\partial_{\lambda} X_{w}\right)\right) \longrightarrow H^{0}\left(F_{w}, q_{2}^{*} L_{\lambda}\left(-\partial_{\lambda} X_{w}\right)\right)
$$

Proposition 4. (1) These maps are isomorphisms, and the restriction map

$$
H^{0}\left(F_{w}, q_{2}^{*} L_{\lambda}\left(-\partial_{\lambda} X_{w}\right)\right) \longrightarrow H^{0}\left(F_{w}^{v}, q_{2}^{*} L_{\lambda}\left(-\left(\partial_{\lambda} X_{w}\right)^{v}\right)\right)
$$

is surjective.

(2) The ascending filtration of $\mathcal{O}_{F_{w}}$ yields an ascending filtration of the $T$ module $H^{0}\left(F_{w}, q_{2}^{*} L_{\lambda}\left(-\partial_{\lambda} X_{w}\right)\right)$, with associated graded

$$
\bigoplus_{x \in W^{P}, x \leq w} H^{0}\left(X_{w}^{x}, L_{\lambda}\left(-\left(\partial_{\lambda} X_{w}\right)^{x}-\left(\partial X^{x}\right)_{w}\right)\right) .
$$

(3) The image of this filtration under restriction to $F_{w}^{v}$ has associated graded

$$
\bigoplus_{x \in W^{P}, v \leq x \leq w} H^{0}\left(X_{w}^{x}, L_{\lambda}\left(-\left(\partial_{\lambda} X_{w}\right)^{x}-\left(\partial X^{x}\right)_{w}\right)\right) .
$$

Hence this is also the associated graded of an ascending filtration

$$
H^{0}\left(X_{w}^{v}, L_{\lambda}\left(-\left(\partial_{\lambda} X_{w}\right)^{v}\right)\right)_{\leq_{t} x}, v \leq x \leq w
$$

of $H^{0}\left(X_{w}^{v}, L_{\lambda}\left(-\left(\partial_{\lambda} X_{w}\right)^{v}\right)\right)$, compatible with the $T$-action and with restrictions to smaller Richardson varieties. 
(4) The subspace

$$
H^{0}\left(X_{w}^{v}, L_{\lambda}\left(-\left(\partial_{\lambda} X_{w}\right)^{v}\right)\right)_{\leq_{t} x} \subseteq H^{0}\left(X_{w}^{v}, L_{\lambda}\right)
$$

consists of those sections that vanish identically on $\left(\partial_{\lambda} X_{w}\right)^{v}$ and on $X_{w}^{y}$ for all $y>_{t} x$. Further, the map

$$
\begin{aligned}
H^{0}\left(X_{w}^{v}, L_{\lambda}\left(-\left(\partial_{\lambda} X_{w}\right)^{v}\right)\right)_{\leq_{t} x} \longrightarrow \operatorname{gr}_{x} H^{0}\left(X_{w}^{v}, L_{\lambda}\left(-\left(\partial_{\lambda} X_{w}\right)^{v}\right)\right) & \\
& =H^{0}\left(X_{w}^{x}, L_{\lambda}\left(-\left(\partial_{\lambda} X_{w}\right)^{x}-\left(\partial X^{x}\right)_{w}\right)\right)
\end{aligned}
$$

is just the restriction to $X_{w}^{x}$.

Proof. It follows from Lemma 7 that the sheaf $q_{2}^{*} L_{\lambda}\left(-\left(\partial_{\lambda} X_{w}\right)^{v}\right)$ on $F_{w}^{v}$ admits a descending filtration with associated graded

$$
\left.\bigoplus_{x \in W^{P}, v \leq x \leq w} \mathcal{O}_{X_{x}^{v}}\left(-\left(\partial X_{x}\right)^{v}\right) \otimes L_{\lambda}\right|_{X_{w}^{x}}\left(-\left(\partial_{\lambda} X_{w}\right)^{v}\right),
$$

and an ascending filtration with associated graded

$$
\left.\bigoplus_{x \in W^{P}, v \leq x \leq w} \mathcal{O}_{X_{x}^{v}} \otimes L_{\lambda}\right|_{X_{w}^{x}}\left(-\left(\partial_{\lambda} X_{w}\right)^{v}-\left(\partial X_{x}\right)^{v}\right) .
$$

As in the proof of Proposition 3 the associated graded of the first filtration is acyclic for $q_{2 *}$; it follows that the adjunction map is an isomorphism. Further, the restriction map

$$
H^{0}\left(X_{w}, L_{\lambda}\left(-\partial_{\lambda} X_{w}\right)\right) \longrightarrow H^{0}\left(X_{w}^{v}, L_{\lambda}\left(-\left(\partial_{\lambda} X_{w}\right)^{v}\right)\right)
$$

is surjective by Corollary 1 Finally, the associated graded of the second filtration is acyclic by Proposition 2. These facts imply our statements, as in the proof of Proposition 3 .

Remarks. (1) By Proposition [3, the $H^{0}\left(G / P, L_{\lambda}\right)_{\leq_{t} x}$ are $B^{-}$-submodules of $H^{0}\left(G / P, L_{\lambda}\right)$. Likewise, the descending filtration of $\mathcal{O}_{F}$ yields a descending filtration of $H^{0}\left(G / P, L_{\lambda}\right)$ by $B$-submodules $H^{0}\left(G / P, L_{\lambda}\right)_{\geq_{t} x}$, consisting of those sections that vanish on $X_{y}$ whenever $y<_{t} x$.

(2) We may have defined directly the preceding filtrations by Propositions [3 (4) and 4 (4), without using the degeneration of the diagonal constructed in Lemma 7. In fact, this alternative definition suffices for the construction of a standard basis in the next section. But the degeneration of the diagonal will play an essential rôle in the section on standard products.

\section{Construction of a standard Basis}

In this section, we fix a dominant weight $\lambda$ and we consider Richardson varieties in $G / P$, where $P=P_{\lambda}$. We shall construct a basis of $H^{0}\left(G / P, L_{\lambda}\right)$ adapted to the filtrations of Propositions 3 and 4 We first prove the key

Lemma 8. Let $v \leq w \in W^{\lambda}$. Then any element of $H^{0}\left(X_{w}^{v}, L_{\lambda}\left(-\left(\partial X_{w}\right)^{v-}\right.\right.$ $\left.\left.\left(\partial X^{v}\right)_{w}\right)\right)$ can be lifted to an element of $H^{0}\left(G / P, L_{\lambda}\right)$ that vanishes identically on all Schubert varieties $X_{y}, y \nsupseteq w$, and on all opposite Schubert varieties $X^{x}, x \nless \leq$.

Proof. Put

$$
X=X_{w}^{v} \text { and } Y=\left(\bigcup_{y \nsupseteq w} X_{y}\right) \cup\left(\bigcup_{x \not z v} X^{x}\right) .
$$


Notice that

$$
X \cap Y=\left(\partial X_{w}\right)^{v} \cup\left(\partial X^{v}\right)_{w}
$$

(as schemes), since any intersection of unions of Richardson varieties is reduced. This yields an exact sequence

$$
0 \longrightarrow \mathcal{I}_{X \cup Y} \longrightarrow \mathcal{I}_{Y} \longrightarrow \mathcal{I}_{Y} \otimes_{\mathcal{O}_{G / P}} \mathcal{O}_{X} \simeq \mathcal{O}_{X}(-X \cap Y) \longrightarrow 0
$$

Tensoring by $L_{\lambda}$ and taking the associated long exact sequence of cohomology groups yields an exact sequence

$$
H^{0}\left(G / P, L_{\lambda} \otimes \mathcal{I}_{Y}\right) \longrightarrow H^{0}\left(X, L_{\lambda}(-X \cap Y)\right) \longrightarrow H^{1}\left(X, L_{\lambda} \otimes \mathcal{I}_{X \cup Y}\right) .
$$

Further, $H^{1}\left(X, L_{\lambda} \otimes \mathcal{I}_{X \cup Y}\right)=0$ by Lemma 5 this completes the proof.

Definition 4. For any $v \leq w \in W^{\lambda}$, let

$$
H_{w}^{v}(\lambda)=H^{0}\left(X_{w}^{v}, L_{\lambda}\left(-\left(\partial X_{w}\right)^{v}-\left(\partial X^{v}\right)_{w}\right)\right)
$$

and

$$
\chi_{w}^{v}(\lambda)=\left\{\text { the weights of the } T \text {-module } H_{w}^{v}(\lambda)\right\},
$$

these weights being counted with multiplicity. Let

$$
\left\{p_{w, v}^{\xi}, \xi \in \chi_{w}^{v}(\lambda)\right\}
$$

be a basis for $H_{w}^{v}(\lambda)$, where each $p_{w, v}^{\xi}$ is a $T$-eigenvector of weight $\xi$.

For any triple $(w, v, \xi)$ as above, let $p_{\pi}$ be a lift of $p_{w, v}^{\xi}$ in $H^{0}\left(G / P, L_{\lambda}\right)$ such that:

$p_{\pi}$ is a $T$-eigenvector of weight $\xi$, and

$p_{\pi}$ vanishes identically on all $X_{y}, y \nsupseteq w$ and on all $X^{x}, x \not \leq v$.

(The existence of such lifts follows from Lemma 8) If $v=w$, then $X_{w}^{v}$ consists of the point $e_{w}$, and hence $\chi_{w}^{v}(\lambda)$ consists of the weight $-w(\lambda)$. We then denote the unique $p_{w, v}^{\xi}$ by $p_{w}$. Its lift to $H^{0}\left(G / P, L_{\lambda}\right)$ is unique; it is the extremal weight vector $p_{w}$.

Definition 5. Let $\pi=(w, v, \xi)$ be as in Definition 4. We set $i(\pi)=w, e(\pi)=v$, and call them respectively the initial and end elements of $\pi$.

By construction of the $p_{\pi}$ and Lemma 1 we obtain:

Lemma 9. With notation as above, we have for $x, y \in W^{\lambda}$,

$$
\left.p_{\pi}\right|_{X_{y}^{x}} \neq 0 \Longleftrightarrow X_{i(\pi)}^{e(\pi)} \subseteq X_{y}^{x} \Longleftrightarrow x \leq e(\pi) \leq i(\pi) \leq y .
$$

Proposition 5. The restrictions to $X_{v}^{w}$ of the $p_{\pi}$ where $i(\pi)=w, e(\pi) \geq v$ form a basis for the $T$-module $H^{0}\left(X_{w}^{v}, L_{\lambda}\left(-\left(\partial X_{w}\right)^{v}\right)\right)$, adapted to its ascending filtration $\leq_{t}$ of Proposition 4 .

Proof. By construction, $p_{\pi}$ vanishes identically on $X^{x}$ for any $x \not \leq e(\pi)$, and hence for any $x>_{t} e(\pi)$. Thus, $p_{\pi} \in H^{0}\left(G / P, L_{\lambda}\right)_{\leq_{t} e(\pi)}$ by Proposition 3 Further, the image of $p_{\pi}$ in the associated graded is just its restriction to $X^{e(\pi)}$.

Together with Lemma 9, it follows that the restrictions of the $p_{\pi}$ to $X_{w}^{v}$ belong to $H^{0}\left(X_{w}^{v}, L_{\lambda}\left(-\left(\partial X_{w}\right)^{v}\right)\right)_{\leq_{t} e(\pi)}$, and that their images in the associated graded $H_{w}^{e(\pi)}(\lambda)$ are the restrictions of the $p_{\pi}$ to $X_{w}^{e(\pi)}$; by construction, these images form a basis of $H_{w}^{e(\pi)}(\lambda)$. 
Now the $T$-module $H^{0}\left(X_{w}^{v}, L_{\lambda}\right)$ has a descending filtration by the submodules

$$
H^{0}\left(X_{w}^{v}, L_{\lambda}\left(-\left(\partial X_{w}\right)^{v}\right)\right)_{\geq_{t} x}
$$

consisting of those sections that vanish identically on $X_{y}^{v}$ whenever $y<_{t} x$. Also, as in Proposition 3, the associated graded is

$$
\bigoplus_{x \in W^{P}, v \leq x \leq w} H^{0}\left(X_{x}^{v}, L_{\lambda}\left(-\left(\partial X_{x}\right)^{v}\right)\right)
$$

Further, we may check as in the proof of Proposition [ 5 that

$$
\left.p_{\pi}\right|_{X_{w}^{v}} \in H^{0}\left(X_{w}^{v}, L_{\lambda}\left(-\left(\partial X_{w}\right)^{v}\right)\right)_{\geq_{t} i(\pi)}
$$

whenever $i(\pi) \geq w$, and the image of $p_{\pi}$ in the associated graded is just its restriction to $X_{i(\pi)}^{v}$. Together with Proposition 5, this implies

Proposition 6. The restrictions to $X_{w}^{v}$ of the $p_{\pi}$ where $v \leq e(\pi) \leq i(\pi) \leq w$ form a basis of $H^{0}\left(X_{w}^{v}, L_{\lambda}\right)$; the $p_{\pi}$ where $v \not \leq e(\pi)$ or $i(\pi) \not \leq w$ form a basis of the kernel of the restriction map $H^{0}\left(G / P, L_{\lambda}\right) \longrightarrow H^{0}\left(X_{w}^{v}, L_{\lambda}\right)$.

In view of Proposition [6, the restriction to $p_{\pi}$ to $X_{y}^{x}$, where $x \leq e(\pi) \leq i(\pi) \leq y$, will be denoted by just $p_{\pi}$.

Definition 6. Set

$$
\Pi(\lambda)=\left\{(v, w, \xi) \mid v, w \in W^{\lambda}, v \leq w, \xi \in \chi_{w}^{v}(\lambda)\right\} .
$$

For any $v, w \in W^{\lambda}, v \leq w$, set

$$
\Pi_{w}^{v}(\lambda):=\{\pi \mid v \leq e(\pi) \leq i(\pi) \leq w\} .
$$

In view of Lemma 9, we have, $\Pi_{w}^{v}(\lambda)=\left\{\pi \in \Pi(\lambda)\left|p_{\pi}\right|_{X_{w}^{v}} \neq 0\right\}$.

More generally, for a union $Z$ of Richardson varieties, define

$$
\Pi_{Z}(\lambda)=\left\{\pi \in \Pi(\lambda)\left|p_{\pi}\right|_{Z} \neq 0\right\} .
$$

Theorem 1. Let $Z$ be a union of Richardson varieties. Then $\left\{\left.p_{\pi}\right|_{Z}, \pi \in \Pi_{Z}(\lambda)\right\}$ is a basis for $H^{0}\left(Z, L_{\lambda}\right)$, and $\left\{p_{\pi}, \pi \in \Pi(\lambda)-\Pi_{Z}(\lambda)\right\}$ is a basis for the kernel of the restriction map $H^{0}\left(G / P, L_{\lambda}\right) \longrightarrow H^{0}\left(Z, L_{\lambda}\right)$.

Proof. By definition of $\Pi_{Z}(\lambda)$, it suffices to prove the first assertion. Let $Z=$ $\bigcup_{i=1}^{r} X_{w_{i}}^{v_{i}}$. We shall prove the result by induction on $r$ and $\operatorname{dim} Z$. Write $Z=X \cup Y$ where $X=X_{w_{i}}^{v_{i}}$ for some $i$, and $\operatorname{dim} X=\operatorname{dim} Z$. Then $X \cap Y$ is a union of Richardson varieties of dimension $<\operatorname{dim} Z$. Consider the exact sequence

$$
0 \rightarrow \mathcal{O}_{Z}=\mathcal{O}_{X \cup Y} \rightarrow \mathcal{O}_{X} \oplus \mathcal{O}_{Y} \rightarrow \mathcal{O}_{X \cap Y} \rightarrow 0 .
$$

Tensoring by $L_{\lambda}$, taking global sections and using the vanishing of $H^{1}\left(Z, L_{\lambda}\right)$ (Lemma 5), we obtain the exact sequence

$$
0 \longrightarrow H^{0}\left(Z, L_{\lambda}\right) \longrightarrow H^{0}\left(X, L_{\lambda}\right) \oplus H^{0}\left(Y, L_{\lambda}\right) \longrightarrow H^{0}\left(X \cap Y, L_{\lambda}\right) \longrightarrow 0 .
$$

In particular, denoting $\operatorname{dim} H^{0}\left(Z, L_{\lambda}\right)$ by $h^{0}\left(Z, L_{\lambda}\right)$ etc., we obtain,

$$
h^{0}\left(Z, L_{\lambda}\right)=h^{0}\left(X, L_{\lambda}\right)+h^{0}\left(Y, L_{\lambda}\right)-h^{0}\left(X \cap Y, L_{\lambda}\right) .
$$

We have by hypothesis (and induction hypothesis), $h^{0}\left(X, L_{\lambda}\right)=\# \Pi_{X}(\lambda), h^{0}\left(Y, L_{\lambda}\right)$ $=\# \Pi_{Y}(\lambda), h^{0}\left(X \cap Y, L_{\lambda}\right)=\# \Pi_{X \cap Y}(\lambda)$. Thus we obtain,

$$
h^{0}\left(Z, L_{\lambda}\right)=\# \Pi_{X}(\lambda)+\# \Pi_{Y}(\lambda)-\# \Pi_{X \cap Y}(\lambda) .
$$


On the other hand, we have

$$
\Pi_{Z}(\lambda)=\left(\Pi_{X}(\lambda) \dot{\cup} \Pi_{Y}(\lambda)\right) \backslash \Pi_{X \cap Y}(\lambda) .
$$

From (1) and (2), we obtain, $h^{0}\left(Z, L_{\lambda}\right)=\# \Pi_{Z}(\lambda)$. Further, the $\left.p_{\pi}\right|_{Z}, \pi \in \Pi_{Z}(\lambda)$, span $H^{0}\left(Z, L_{\lambda}\right)$ (since the $p_{\pi}, \pi \in \Pi(\lambda)$, span $H^{0}\left(G / P, L_{\lambda}\right)$, and the restriction map $H^{0}\left(G / P, L_{\lambda}\right) \rightarrow H^{0}\left(Z, L_{\lambda}\right)$ is surjective). Thus, the $\left.p_{\pi}\right|_{Z}, \pi \in \Pi_{Z}(\lambda)$, are a basis of $H^{0}\left(Z, L_{\lambda}\right)$.

\section{STANDARD MONOMIALS}

Let $\lambda, \mu$ be dominant weights such that $P_{\lambda}=P_{\mu}:=P$. Consider the product map

$$
H^{0}\left(G / P, L_{\lambda}\right) \otimes H^{0}\left(G / P, L_{\mu}\right) \longrightarrow H^{0}\left(G / P, L_{\lambda+\mu}\right) .
$$

This map is surjective by [16] 2.2 and 3.5. Using Proposition 1 , it follows that the product map

$$
H^{0}\left(X_{w}^{v}, L_{\lambda}\right) \otimes H^{0}\left(X_{w}^{v}, L_{\mu}\right) \longrightarrow H^{0}\left(X_{w}^{v}, L_{\lambda+\mu}\right)
$$

is also surjective, for any $v \leq w$ in $W^{P}$. We shall construct a basis for $H^{0}\left(X_{w}^{v}, L_{\lambda+\mu}\right)$ from the bases of $H^{0}\left(X_{w}^{v}, L_{\lambda}\right), H^{0}\left(X_{w}^{v}, L_{\mu}\right)$ obtained in Theorem [1. For this, we need the following

Definition 7. Let $v, w \in W^{P}, v \leq w$. Let $\varphi \in \Pi_{w}^{v}(\lambda)$ and $\psi \in \Pi_{w}^{v}(\mu)$. The pair $(\varphi, \psi)$ is called standard on $X_{w}^{v}$ if

$$
v \leq e(\psi) \leq i(\psi) \leq e(\varphi) \leq i(\varphi) \leq w .
$$

Then the product $p_{\varphi} p_{\psi} \in H^{0}\left(G / P, L_{\lambda+\mu}\right)$ is called standard on $X_{w}^{v}$ as well.

Clearly, we have

Lemma 10. Let $p_{\varphi} p_{\psi}$ be a standard product on $G / P$ and let $v \leq w \in W^{P}$. Then

$$
\left.p_{\varphi} p_{\psi}\right|_{X_{w}^{v}} \neq 0 \Longleftrightarrow v \leq e(\psi) \leq i(\varphi) \leq w .
$$

Proposition 7. The standard products on $X_{w}^{v}$ form a basis of $H^{0}\left(X_{w}^{v}, L_{\lambda+\mu}\right)$. The standard products on $G / P$ that are not standard on $X_{w}^{v}$ form a basis of the kernel of the restriction map $H^{0}\left(G / P, L_{\lambda+\mu}\right) \longrightarrow H^{0}\left(X_{w}^{v}, L_{\lambda+\mu}\right)$.

Proof. Consider the $T$-linearized invertible sheaf $q_{1}^{*} L_{\mu} \otimes q_{2}^{*} L_{\lambda}$ on $F_{w}^{v}$. By Lemma (7) the ascending filtration of $\mathcal{O}_{F_{w}^{v}}$ yields one of that sheaf, with associated graded

$$
\left.\left.\bigoplus_{x \in W^{P}, v \leq x \leq w} L_{\mu}\right|_{X_{x}^{v}}\left(-\left(\partial X_{x}\right)^{v}\right) \otimes L_{\lambda}\right|_{X_{w}^{x}} .
$$

By Proposition 1, the latter sheaf is acyclic. This yields an ascending filtration of the $T$-module $H^{0}\left(F_{w}^{v}, q_{1}^{*} L_{\mu} \otimes q_{2}^{*} L_{\lambda}\right)$, with associated graded

$$
\bigoplus_{x \in W^{P}, v \leq x \leq w} H^{0}\left(X_{x}^{v}, L_{\mu}\left(-\left(\partial X_{x}\right)^{v}\right)\right) \otimes H^{0}\left(X_{w}^{x}, L_{\lambda}\right) ;
$$

it also follows that $H^{i}\left(F_{w}^{v}, q_{1}^{*} L_{\mu} \otimes q_{2}^{*} L_{\lambda}\right)=0$ for all $i \geq 1$.

By Proposition 3 we may identify $H^{0}\left(X_{w}^{v}, L_{\lambda}\right)$ with $H^{0}\left(F_{w}^{v}, q_{2}^{*} L_{\lambda}\right)$; likewise, we may identify $H^{0}\left(X_{w}^{v}, L_{\mu}\right)$ with $H^{0}\left(F_{w}^{v}, q_{1}^{*} L_{\mu}\right)$. Using the multiplication map

$$
H^{0}\left(F_{w}^{v}, q_{1}^{*} L_{\mu}\right) \otimes H^{0}\left(F_{w}^{v}, q_{2}^{*} L_{\lambda}\right) \longrightarrow H^{0}\left(F_{w}^{v}, q_{1}^{*} L_{\mu} \otimes q_{2}^{*} L_{\lambda}\right),
$$

this defines "dot products" in $H^{0}\left(F_{w}^{v}, q_{1}^{*} L_{\mu} \otimes q_{2}^{*} L_{\lambda}\right)$. 
Let $x \in W^{P}$ such that $v \leq x \leq w$. Recall that the $p_{\psi}, v \leq e(\psi) \leq i(\psi)=x$, are a basis of $H^{0}\left(X_{x}^{v}, L_{\mu}\left(-\left(\partial X_{x}\right)^{v}\right)\right)$. Further, the $p_{\varphi}, x \leq e(\varphi) \leq i(\varphi) \leq w$, are a basis of $H^{0}\left(X_{w}^{x}, L_{\lambda}\right)$. Thus, the dot products $p_{\psi} \cdot p_{\varphi}$, where there exists $x \in W^{P}$ such that

$$
v \leq e(\psi) \leq i(\psi)=x \text { and } x \leq e(\varphi) \leq i(\varphi) \leq w,
$$

restrict to a basis of $H^{0}\left(X_{x}^{v}, L_{\mu}\left(-\left(\partial X_{x}\right)^{v}\right)\right) \otimes H^{0}\left(X_{w}^{x}, L_{\lambda}\right)$. By construction of the filtration of $H^{0}\left(F_{w}^{v}, q_{1}^{*} L_{\mu} \otimes q_{2}^{*} L_{\lambda}\right)$, it follows that the standard dot products are a basis of that space.

Consider now the $T$-linearized invertible sheaf $p_{1}^{*} L_{\mu} \otimes p_{2}^{*} L_{\lambda}$ on $\mathcal{X}_{w}^{v}$. This sheaf is flat on $\mathbb{A}^{1}$; by vanishing of $H^{1}\left(F_{w}^{v}, q_{1}^{*} L_{\mu} \otimes q_{2}^{*} L_{\lambda}\right)$ and semicontinuity, it follows that the restriction

$$
H^{0}\left(\mathcal{X}_{w}^{v}, p_{1}^{*} L_{\mu} \otimes p_{2}^{*} L_{\lambda}\right) \longrightarrow H^{0}\left(F_{w}^{v}, q_{1}^{*} L_{\mu} \otimes q_{2}^{*} L_{\lambda}\right)
$$

is surjective, and that $H^{0}\left(\mathcal{X}_{w}^{v}, p_{1}^{*} L_{\mu} \otimes p_{2}^{*} L_{\lambda}\right)$ is a free module over $H^{0}\left(\mathbb{A}^{1}, \mathcal{O}_{\mathbb{A}_{1}}\right)=$ $k[z]$, generated by any lift of its quotient space $H^{0}\left(F_{w}^{v}, q_{1}^{*} L_{\mu} \otimes q_{2}^{*} L_{\lambda}\right)$.

We now construct such a lift, as follows. Consider the adjunction maps

$$
H^{0}\left(X_{w}^{v}, L_{\lambda}\right) \longrightarrow H^{0}\left(\mathcal{X}_{w}^{v}, p_{2}^{*} L_{\lambda}\right) \text { and } H^{0}\left(X_{w}^{v}, L_{\mu}\right) \longrightarrow H^{0}\left(\mathcal{X}_{w}^{v}, p_{1}^{*} L_{\mu}\right)
$$

These yield dot products $p_{\psi} \cdot p_{\varphi}$ in $H^{0}\left(\mathcal{X}_{w}^{v}, p_{1}^{*} L_{\mu} \otimes p_{2}^{*} L_{\lambda}\right)$ which lift the corresponding products in $H^{0}\left(F_{w}^{v}, q_{1}^{*} L_{\mu} \otimes q_{2}^{*} L_{\lambda}\right)$. Since the latter standard products are a basis of that space, the standard dot products $p_{\psi} \cdot p_{\varphi}$ are a basis of $H^{0}\left(\mathcal{X}_{w}^{v}, p_{1}^{*} L_{\mu} \otimes p_{2}^{*} L_{\lambda}\right)$ over $k[z]$. Therefore, they restrict to a basis of the space of sections of $p_{1}^{*} L_{\mu} \otimes p_{2}^{*} L_{\lambda}$ over any fiber of $\pi$. But the fiber at 1 is $\operatorname{diag}\left(X_{w}^{v}\right)$, and the restriction of $p_{1}^{*} L_{\mu} \otimes p_{2}^{*} L_{\lambda}$ to that fiber is just $L_{\lambda+\mu}$ whereas the restrictions of the dot products are just the usual products. We have proved that the standard products on $X_{w}^{v}$ form a basis of $H^{0}\left(X_{w}^{v}, L_{\lambda+\mu}\right)$.

To complete the proof, notice that any standard product on $G / P$ that is not standard on $X_{w}^{v}$ vanishes identically on that subvariety by Lemma 10.

Remark. The proof of Proposition 7 relies on the fact that the special fiber $F_{w}^{v}$ of the flat family $\pi: \mathcal{X}_{w}^{v} \rightarrow \mathbb{A}^{1}$ equals $\bigcup_{x \in W^{P}, v \leq x \leq w} X_{x}^{v} \times X_{w}^{x}$. Conversely, this fact can be recovered from Proposition 7 as follows.

We have the equalities of Euler characteristics:

$$
\chi\left(F, q_{1}^{*} L_{\mu} \otimes q_{2}^{*} L_{\lambda}\right)=\chi\left(G / P, L_{\lambda+\mu}\right)=\sum_{x \in W^{P}} \chi\left(X_{x}, L_{\mu}\left(-\partial X_{x}\right)\right) \chi\left(X^{x}, L_{\lambda}\right),
$$

where the first equality holds by flatness of $\pi$, and the second one by Propositions 5 6] and 7 . It follows that

$$
\chi\left(F, q_{1}^{*} L_{\mu} \otimes q_{2}^{*} L_{\lambda}\right)=\chi\left(\bigcup_{x \in W^{P}} X_{x} \times X^{x}, q_{1}^{*} L_{\mu} \otimes q_{2}^{*} L_{\lambda}\right) .
$$

Since $F$ contains $\bigcup_{x \in W^{P}} X_{x} \times X^{x}$ by the first claim in the proof of Lemma 7 and $\lambda$, $\mu$ are arbitrary dominant $P$-regular weights, it follows that $F=\bigcup_{x \in W^{P}} X_{x} \times X^{x}$ (e.g., since both have the same Hilbert polynomial). Now the argument of Lemma 7 yields $F_{w}^{v}=\bigcup_{x \in W^{P}, v \leq x \leq w} X_{x}^{v} \times X_{w}^{x}$.

We now extend Proposition 7 to unions of Richardson varieties. 
Definition 8. Let $\Pi(\lambda, \mu)$ be the set of all standard pairs $(\varphi, \psi)$ where $\varphi \in \Pi(\lambda)$ and $\psi \in \Pi(\mu)$. For $v \leq w \in W^{P}$, let $\Pi_{w}^{v}(\lambda, \mu)$ be the subset of standard pairs on $X_{w}^{v}$. In view of Lemma 10, we have

$$
\Pi_{w}^{v}(\lambda, \mu)=\left\{(\varphi, \psi) \in \Pi(\lambda, \mu)\left|p_{\varphi} p_{\psi}\right|_{X_{w}^{v}} \neq 0\right\} .
$$

Finally, for a union $Z$ of Richardson varieties, let

$$
\Pi_{Z}(\lambda, \mu)=\left\{(\varphi, \psi) \in \Pi(\lambda, \mu)\left|p_{\varphi} p_{\psi}\right|_{Z} \neq 0\right\} .
$$

Now arguing as in the proof of Theorem 1 we obtain

Theorem 2. Let $Z$ be a union of Richardson varieties in $G / P$. Then the products $p_{\varphi} p_{\psi}$, where $(\varphi, \psi) \in \Pi_{Z}(\lambda, \mu)$, form a basis of $H^{0}\left(Z, L_{\lambda+\mu}\right)$. The products $p_{\varphi} p_{\psi}$, where $(\varphi, \psi) \in \Pi(\lambda, \mu)-\Pi_{Z}(\lambda, \mu)$, form a basis of the kernel of the restriction map $H^{0}\left(G / P, L_{\lambda+\mu}\right) \longrightarrow H^{0}\left(Z, L_{\lambda+\mu}\right)$.

Corollary 2. For any $\varphi \in \Pi(\lambda)$ and $\psi \in \Pi(\mu)$, the product $p_{\varphi} p_{\psi} \in H^{0}\left(G / P, L_{\lambda+\mu}\right)$ is a linear combination of standard products $p_{\varphi^{\prime}} p_{\psi^{\prime}}$ where $i\left(\varphi^{\prime}\right) \geq i(\varphi)$ and $e\left(\psi^{\prime}\right) \leq$ $e(\psi)$.

Proof. Notice that $p_{\varphi} p_{\psi}$ vanishes identically on all $X_{y}$ where $y \geq i(\varphi)$, and on all $X^{x}$ where $x \not \leq e(\psi)$. By Theorem 2, it follows that $p_{\varphi} p_{\psi}$ is a linear combination of standard products $p_{\varphi^{\prime}} p_{\psi^{\prime}}$, where $i(\varphi)^{\prime} \not \leq y$ whenever $y \geq i(\varphi)$, and $e(\psi)^{\prime} \geq x$ whenever $x \not \leq e(\psi)$. But this means exactly that $i\left(\varphi^{\prime}\right) \geq i(\varphi)$ and $e\left(\psi^{\prime}\right) \leq e(\psi)$.

Next we consider a family of dominant weights $\lambda_{1}, \ldots, \lambda_{m}$ such that $P=P_{\lambda_{1}}=$ $\cdots=P_{\lambda_{m}}$. For any union $Z$ of Richardson varieties in $G / P$, we shall construct a basis of $H^{0}\left(Z, L_{\lambda_{1}+\cdots+\lambda_{m}}\right)$, in terms of standard monomials of degree $m$. These are defined as follows.

Definition 9. Let $\pi_{i} \in \Pi\left(\lambda_{i}\right)$ for $1 \leq i \leq m$. Then the sequence $\underline{\pi}:=\left(\pi_{1}, \pi_{2}, \ldots, \pi_{m}\right)$ is standard if

$$
e\left(\pi_{m}\right) \leq i\left(\pi_{m}\right) \leq \cdots \leq e\left(\pi_{1}\right) \leq i\left(\pi_{1}\right) .
$$

Further, let $v, w \in W^{P}$ such that $v \leq w$; then $\underline{\pi}$ is standard on $X_{w}^{v}$ if

$$
v \leq e\left(\pi_{m}\right) \leq i\left(\pi_{m}\right) \leq \cdots \leq e\left(\pi_{1}\right) \leq i\left(\pi_{1}\right) \leq w .
$$

Finally, $\underline{\pi}$ is standard on $Z=\bigcup X_{w_{i}}^{v_{i}}$ if it is standard on $X_{w_{i}}^{v_{i}}$ for some $i$.

Set

$$
\begin{gathered}
\Pi_{w}^{v}\left(\lambda_{1}, \ldots, \lambda_{m}\right)=\left\{\underline{\pi}=\left(\pi_{1}, \pi_{2}, \ldots, \pi_{m}\right) \mid \underline{\pi} \text { is standard on } X_{w}^{v}\right\}, \\
\Pi_{Z}\left(\lambda_{1}, \ldots, \lambda_{m}\right)=\left\{\underline{\pi}=\left(\pi_{1}, \pi_{2}, \ldots, \pi_{m}\right) \mid \underline{\pi} \text { is standard on } Z\right\} .
\end{gathered}
$$

Definition 10. Given $\underline{\pi}=\left(\pi_{1}, \pi_{2}, \ldots, \pi_{m}\right)$, set $p_{\underline{\pi}}:=p_{\pi_{1}} \cdots p_{\pi_{m}}$.

Note that $p_{\underline{\pi}} \in H^{0}\left(G / P, L_{\lambda_{1}+\cdots+\lambda_{m}}\right)$. If $\underline{\pi}$ is standard, then we call $p_{\underline{\underline{\pi}}}$ a standard monomial on $G / P$. If $\underline{\pi}$ is standard on $X_{w}^{v}$ (resp. $Z$ ), then we call $p_{\underline{\pi}}$ a standard monomial on $X_{w}^{v}$ (resp. $Z$ ).

By Theorem 2 and induction on $m$, we obtain

Corollary 3. Let $Z$ be a union of Richardson varieties in $G / P$ and let $\lambda_{1}, \ldots, \lambda_{m}$ be dominant weights such that $P=P_{\lambda_{1}}=\cdots=P_{\lambda_{m}}$. Then the monomials $p_{\underline{\pi}}$ where $\underline{\pi} \in \Pi_{Z}\left(\lambda_{1}, \ldots, \lambda_{m}\right)$ form a basis of $H^{0}\left(Z, L_{\lambda_{1}+\cdots+\lambda_{m}}\right)$. Further, the monomials $p_{\underline{\pi}}$ where $\underline{\pi} \in \Pi\left(\lambda_{1}, \ldots, \lambda_{m}\right)-\Pi_{Z}\left(\lambda_{1}, \ldots, \lambda_{m}\right)$, form a basis of the kernel of the restriction map $H^{0}\left(G / P, L_{\lambda_{1}+\cdots+\lambda_{m}}\right) \longrightarrow H^{0}\left(Z, L_{\lambda_{1}+\cdots+\lambda_{m}}\right)$. 
As an application, we determine the equations of unions of Richardson varieties in their projective embeddings given by very ample line bundles on $G / P$. Let $\lambda$ be a dominant $P$-regular weight. For any $\pi_{1}, \pi_{2} \in \Pi(\lambda)$, we have in $H^{0}\left(G / P, L_{2 \lambda}\right)$ :

$$
p_{\pi_{1}} p_{\pi_{2}}-\sum a_{\pi_{1}^{\prime}, \pi_{2}^{\prime}} p_{\pi_{1}^{\prime}} p_{\pi_{2}^{\prime}}=0
$$

where $a_{\pi_{1}^{\prime}, \pi_{2}^{\prime}} \in k$ and the sum is over those standard pairs $\left(\pi_{1}^{\prime}, \pi_{2}^{\prime}\right) \in \Pi(\lambda, \lambda)$ such that $i\left(\pi_{1}^{\prime}\right) \geq i\left(\pi_{1}\right)$ and $e\left(\pi_{2}^{\prime}\right) \leq e\left(\pi_{2}\right)$ (as follows from Corollary 2).

Definition 11. The preceding elements $p_{\pi_{1}} p_{\pi_{2}}-\sum a_{\pi_{1}^{\prime}, \pi_{2}^{\prime}} p_{\pi_{1}^{\prime}} p_{\pi_{2}^{\prime}}$ when regarded in $S^{2} H^{0}\left(G / P, L_{\lambda}\right)$, will be called the quadratic straightening relations.

Corollary 4. Let $\lambda$ be a regular dominant character of $P$.

(1) The multiplication map

$$
\bigoplus_{m=0}^{\infty} S^{m} H^{0}\left(G / P, L_{\lambda}\right) \longrightarrow \bigoplus_{m=0}^{\infty} H^{0}\left(G / P, L_{m \lambda}\right)
$$

is surjective, and its kernel is generated as an ideal by the quadratic straightening relations.

(2) For any union $Z$ of Richardson varieties in $G / P$, the restriction map

$$
\bigoplus_{m=0}^{\infty} H^{0}\left(G / P, L_{m \lambda}\right) \longrightarrow \bigoplus_{m=0}^{\infty} H^{0}\left(Z, L_{m \lambda}\right)
$$

is surjective. Its kernel is generated as an ideal by the $p_{\pi}, \pi \in \Pi(\lambda)-\Pi_{Z}(\lambda)$ together with the standard products $p_{\pi_{1}} p_{\pi_{2}}$ where $i\left(\pi_{1}\right) \not \leq w$ or $e\left(\pi_{2}\right) \nsupseteq v$ whenever $X_{w}^{v}$ is an irreducible component of $Z$. If, in addition, $Z$ is a union of Richardson varieties $X_{w}^{v}$ all having the same $w$, then the $p_{\pi}, \pi \in$ $\Pi(\lambda)-\Pi_{Z}(\lambda)$ suffice.

Proof. (1) By 16], Theorem 3.11, the multiplication map is surjective, and its kernel is generated as an ideal by the kernel $K$ of the map $S^{2} H^{0}\left(G / P, L_{\lambda}\right) \longrightarrow$ $H^{0}\left(G / P, L_{2 \lambda}\right)$. Let $J$ be the subspace of $S^{2} H^{0}\left(G / P, L_{\lambda}\right)$ generated by all quadratic straightening relations. Then $J \subseteq K$, and the quotient space $S^{2} H^{0}\left(G / P, L_{\lambda}\right) / J$ is spanned by the images of the standard products. Further, their images in $S^{2} H^{0}\left(G / P, L_{\lambda}\right) / K \simeq H^{0}\left(G / P, L_{2 \lambda}\right)$ form a basis, by Proposition 7 . It follows that $J=K$.

(2) The first assertion follows from Lemma 5. Consider a standard monomial $p_{\underline{\pi}}=p_{\pi_{1}} \cdots p_{\pi_{m}} \in H^{0}\left(G / P, L_{m \lambda}\right)$. By Corollary [3, $p_{\underline{\pi}}$ vanishes identically on $Z$ if and only if $i\left(\pi_{1}\right) \not \leq w$ or $e\left(\pi_{m}\right) \geq v$ for all irreducible components $X_{w}^{v}$. This amounts to $p_{\pi_{1}} p_{\pi_{m}}$ vanishes identically on $Z$. If, in addition, $w$ is independent of the component, then $p_{\pi_{1}}$ or $p_{\pi_{m}}$ vanishes identically on $Z$; further, $p_{\pi_{1}} p_{\pi_{m}}$ is a standard product on $G / P$. This implies the remaining assertions, since the kernel of $H^{0}\left(G / P, L_{m \lambda}\right) \longrightarrow H^{0}\left(Z, L_{m \lambda}\right)$ is spanned by those standard monomials on $G / P$ that are not standard on $Z$ (Corollary 3 ).

Remark. In particular, the $p_{\pi}$, where $\pi \in \Pi(\lambda)-\Pi_{Z}(\lambda)$, generate the homogeneous ideal of $Z$ in $G / P$, whenever $Z$ is a union of Schubert varieties (or a union of opposite Schubert varieties). But this does not extend to arbitrary unions of Richardson varieties, as shown by the obvious example where $G / P=\mathbb{P}^{1}, Z=\{0, \infty\}$ and $L_{\lambda}=\mathcal{O}(1) ;$ then $\Pi(\lambda)=\Pi_{Z}(\lambda)$. 


\section{Weights OF CLASSICAL TYPE}

In this section, we shall determine the "building blocks"

$$
H_{w}^{v}(\lambda)=H^{0}\left(X_{w}^{v}, L_{\lambda}\left(-\left(\partial X_{w}\right)^{v}-\left(\partial X^{v}\right)_{w}\right)\right)
$$

in the case where the dominant weight $\lambda$ is of classical type (as introduced in [9], cf. the next definition). Along the way, we shall retrieve the results of loc. cit., using our basis $\left\{p_{\pi}\right\}$. In particular, we shall give a geometric characterization of "admissible pairs" of loc. cit. (cf. Definition 16 below).

Definition 12. Let $\lambda$ be a dominant weight. We say, $\lambda$ is of classical type if $\left\langle\lambda, \beta^{\vee}\right\rangle \leq 2$, for all $\beta \in R^{+}$.

Remarks. (1) Any dominant weight of classical type is either fundamental, or a sum of two minuscule fundamental weights.

(2) $G$ is classical if and only if all fundamental weights of $G$ are of classical type.

For the rest of this section, we fix a dominant weight $\lambda$ of classical type.

Proposition 8. Let $v, w \in W^{\lambda}, v \leq w$. Then the $T$-module $H_{w}^{v}(\lambda)$ is at most one-dimensional; further, if nonzero, then it has the weight $-\frac{1}{2}(w(\lambda)+v(\lambda))$.

As a consequence, the weights of the $T$-module $H^{0}\left(X_{w}, L_{\lambda}\left(-\partial X_{w}\right)\right)$ are among the $-\frac{1}{2}(w(\lambda)+x(\lambda))$ where $x \leq w$, and the corresponding weight spaces are onedimensional.

Proof. Let $p \in H_{w}^{v}(\lambda)$. Then $p^{2}$ belongs to $H^{0}\left(X_{w}^{v}, L_{2 \lambda}\right)$, and vanishes of order $\geq 2$ along each component of the whole boundary $\left(\partial X_{w}\right)^{v} \cup\left(\partial X^{v}\right)_{w}$. On the other hand, the product $p_{w} p_{v}$ also belongs to $H^{0}\left(X_{w}^{v}, L_{2 \lambda}\right)$ and satisfies, by Chevalley's formula,

$$
\operatorname{div}\left(p_{w} p_{v}\right)=\sum_{\beta}\left\langle\lambda, \beta^{\vee}\right\rangle X_{w s_{\beta}}^{v}+\sum_{\gamma}\left\langle\lambda, \gamma^{\vee}\right\rangle X_{w}^{v s_{\gamma}},
$$

where $X_{w s_{\beta}}$ (resp. $X^{v s_{\gamma}}$ ) runs over all the components $X_{x}$ (resp. $X^{y}$ ) of $\partial X_{w}$ (resp. $\partial X^{v}$ ) such that $x \geq v$ (resp. $y \leq w$ ). Hence, $p_{w} p_{v}$ vanishes of order at most 2 along each component of $\left(\partial X_{w}\right)^{v} \cup\left(\partial X^{v}\right)_{w}$ (since $\lambda$ is of classical type), and nowhere else. Thus, $\frac{p^{2}}{p_{w} p_{v}}$ (a rational function on $X_{w}^{v}$ ) has no poles. It follows that $p^{2}=c p_{w} p_{v}, c \in k$, and hence that $p$ is unique up to scalars; further, $p$ is either zero or has weight $\frac{1}{2}\left(\right.$ weight $p_{w}+$ weight $\left.p_{v}\right)=-\frac{1}{2}(w(\lambda)+v(\lambda))$.

As a corollary to the proof of the above proposition, we have

Lemma 11. Let $v, w \in W^{\lambda}, v \leq w$. Further, let $H_{w}^{v}(\lambda)$ be nonzero. Then for each divisor $X_{w s_{\beta}}\left(\right.$ resp. $\left.X^{v s_{\gamma}}\right)$ of $X_{w}\left(\right.$ resp. $\left.X^{v}\right)$ such that $w s_{\beta} \geq v\left(\right.$ resp. $\left.v s_{\gamma} \leq w\right)$, $\beta$ (resp. $\gamma)$ being in $R^{+}$, we have, $\left\langle\lambda, \beta^{\vee}\right\rangle\left(\right.$ resp. $\left.\left\langle\lambda, \gamma^{\vee}\right\rangle\right)=2$.

We shall denote by $p_{w, v}$ the unique $p_{w, v}^{\xi}$, if nonzero (then $p_{w, w}=p_{w}$ ). By Proposition $8 p_{w, v}$ lifts to a unique $T$-eigenvector in $H^{0}\left(X_{w}, L_{\lambda}\left(-\partial X_{w}\right)\right)$; we still denote that lift by $p_{w, v}$. The nonzero $p_{w, v}$, where $v \leq w$, form a basis of $H^{0}\left(X_{w}, L_{\lambda}\left(-\partial X_{w}\right)\right)$.

Notice that $\frac{p_{w, v}^{2}}{p_{w}}$ is a rational section of $L_{\lambda}$ on $X_{w}$, eigenvector of $T$ with weight $-v(\lambda)$, and without poles by the argument of Proposition 8 This implies 
Lemma 12. With notation as above, we have $p_{w, v}^{2}=p_{w} p_{v}$ on $X_{w}$, up to a nonzero scalar.

We now aim at characterizing those pairs $(v, w)$ such that $p_{w, v} \neq 0$. For this, we recall some definitions and lemmas from 9 .

Definition 13. Let $X_{v}$ be a Schubert divisor in $X_{w}$; further, let $v=s_{\alpha} w$ where $\alpha \in R^{+}$. If $\alpha$ is simple, then we say, $X_{v}$ is a moving divisor in $X_{w}$, moved by $\alpha$.

Lemma 13 ([9], Lemma 1.5). Let $X_{v}$ be a moving divisor in $X_{w}$, moved by $\alpha$. Let $X_{u}$ be any Schubert subvariety of $X_{w}$. Then either, $X_{u} \subseteq X_{v}$ or $X_{s_{\alpha} u} \subseteq X_{v}$.

Definition 14. Let $v, w \in W^{\lambda}, v \leq w, \ell(v)=\ell(w)-1$; further let $v=w s_{\beta}=s_{\gamma} w$, for some positive roots $\beta, \gamma$. We denote the positive integer $\left\langle\lambda, \beta^{\vee}\right\rangle\left(=\left\langle v(\lambda), \gamma^{\vee}\right\rangle=\right.$ $\left.-\left\langle w(\lambda), \gamma^{\vee}\right\rangle\right)$ by $m_{\lambda}(v, w)$, and refer to it as the Chevalley multiplicity of $X_{v}$ in $X_{w}$ (see [3]).

Lemma 14 (9] Lemma 2.5). Let $v, w \in W^{\lambda}$ such that $X_{v}$ is a moving divisor in $X_{w}$, moved by $\alpha$. Let $X_{u}$ be another Schubert divisor in $X_{w}$. Then $X_{s_{\alpha} u}$ is a divisor in $X_{v}$, and $m_{\lambda}\left(s_{\alpha} u, v\right)=m_{\lambda}(u, w)$.

Definition 15. Let $v, w \in W^{\lambda}$ such that $X_{v}$ is a divisor in $X_{w}$. If $m_{\lambda}(v, w)=2$, then we shall refer to $X_{v}$ as a double divisor in $X_{w}$.

By Lemma 11] if $p_{w, v} \neq 0$, then all Schubert divisors in $X_{w}$ that meet $X^{v}$ are double divisors.

Lemma 15 (9], Lemma 2.6.). Let $u, w \in W^{\lambda}$ such that $X_{u}$ is a double divisor in $X_{w}$. Then $X_{u}$ is a moving divisor in $X_{w}$.

A geometric characterization of Admissible pairs. Recall (cf. 9]):

Definition 16. A pair $(v, w)$ in $W^{\lambda}$ is called admissible if either $v=w$ (in which case, it is called a trivial admissible pair), or there exists a sequence $w=w_{1}>w_{2}>$ $\cdots>w_{r}=v$, such that $X_{w_{i+1}}$ is a double divisor in $X_{w_{i}}$, i.e., $m_{\lambda}\left(w_{i+1}, w_{i}\right)=2$. We shall refer to such a chain as a double chain.

We shall give a geometric characterization of admissible pairs (cf. Proposition 9 below). First we prove some preparatory lemmas.

Lemma 16. Let $p_{w, v} \neq 0$, then

(1) For any double divisor $X_{s_{\alpha} w}$ in $X_{w}$ meeting $X^{v}$, we have

$$
p_{s_{\alpha} w, v}=e_{-\alpha} p_{w, v} \text { and } e_{-\alpha}^{2} p_{w, v}=0,
$$

where $e_{-\alpha}$ is a generator of the Lie algebra of $U_{-\alpha}$. Further, $p_{s_{\alpha} w, v} \neq 0$.

(2) Likewise, for any double divisor $X^{s_{\alpha} v}$ in $X^{v}$ meeting $X_{w}$, we have

$$
p_{w, s_{\alpha} v}=e_{\alpha} p_{w, v} \text { and } e_{\alpha}^{2} p_{w, v}=0,
$$

where $e_{\alpha}$ is a generator of the Lie algebra of $U_{\alpha}$. Further, $p_{w, s_{\alpha} v} \neq 0$.

(3) The pair $(v, w)$ is admissible.

Proof. (1) Consider the $T$-module $H^{0}\left(X_{w}^{v}, L_{\lambda}\left(-\left(\partial X^{v}\right)_{w}\right)\right)$. By Proposition 8, it has a basis $\left\{p_{x, v} \mid v \leq x \leq w\right\}$ with corresponding weights $-\frac{1}{2}(x(\lambda)+v(\lambda))$. Notice that $X_{w}$ is invariant under $U_{-\alpha}$ (since $\left.s_{\alpha} w<w\right)$; hence $X_{w}^{v}$ and $\left(\partial X^{v}\right)_{w}$ are also $U_{-\alpha}$-invariant. Thus, $U_{-\alpha}$ acts on $H^{0}\left(X_{w}^{v}, L_{\lambda}\left(-\left(\partial X^{v}\right)_{w}\right)\right)$, compatibly with the $T$-action. The $U_{-\alpha}$-submodule $M$ generated by $p_{w, v}$ is $T$-invariant, with 
weights of the form $-\frac{1}{2}(w(\lambda)+v(\lambda))-m \alpha$ for some nonnegative integers $m$. But if $x(\lambda)=w(\lambda)+2 m \alpha$, then either $x=w$ and $m=0$, or $x=s_{\alpha} w$ and $m=1$ (by Lemma (11). Hence $M$ is either spanned by $p_{w, v}$, or by $p_{w, v}$ and $p_{s_{\alpha} w, v}$. Further, $e_{-\alpha}^{2} p_{w, v}=0$.

To complete the proof, it suffices to show that $U_{-\alpha}$ does not fix $p_{w, v}$. Otherwise, the zero locus of $p_{w, v}$ in $X_{w}^{v}$ is $U_{-\alpha}$-invariant, and hence so is $\left(\partial X_{w}\right)^{v}$. Thus,

$$
\overline{U_{-\alpha} e_{s_{\alpha w}}} \subseteq\left(\partial X_{w}\right)^{v} .
$$

But $e_{w} \in \overline{U_{-\alpha} e_{s_{\alpha w}}}$ (since $\left.s_{\alpha} w<w\right)$ and $e_{w} \notin\left(\partial X_{w}\right)^{v}$, a contradiction.

(2) is checked similarly. (3) follows from (1) together with Lemma 11 by induction on $\ell(w)$.

Lemma 17. Let $(v, w)$ be an admissible pair, then $p_{w, v} \neq 0$.

Proof. We argue by induction on $\ell(w)$. We may chose a simple root $\alpha$ such that $w>s_{\alpha} w \geq v$ and that $X_{w s_{\alpha}}$ is a double divisor in $X_{w}$. Then $\langle w(\lambda), \check{\alpha}\rangle=-2$, and also $p_{s_{\alpha} w, v} \neq 0$ by the induction hypothesis. The weight of this vector is

$$
-\frac{1}{2}\left(s_{\alpha} w(\lambda)+v(\lambda)\right)=-\frac{1}{2}(w(\lambda)+v(\lambda))-\alpha .
$$

The scalar product of this weight with $\check{\alpha}$ being integral, $\langle v(\lambda), \check{\alpha}\rangle$ is an even integer. Since $\lambda$ is of classical type, it follows that

$$
\langle v(\lambda), \check{\alpha}\rangle \in\{2,0,-2\} .
$$

We now distinguish the following three cases:

Case 1: $\left(v(\lambda), \alpha^{\vee}\right)=2$. Then $w \geq s_{\alpha} w, s_{\alpha} v>v$. As a first step, we find a relation between $H^{0}\left(X_{w}^{v}, L_{\lambda}\left(-\left(\partial X_{w}\right)^{v}\right)\right)$ and $H^{0}\left(X_{s_{\alpha} w}^{v}, L_{\lambda}\left(-\left(\partial X_{s_{\alpha} w}\right)^{v}\right)\right)$.

Let $G_{\alpha}$ be the subgroup of $G$ generated by $U_{\alpha}, U_{-\alpha}$ and $T$; let $B_{\alpha}=G_{\alpha} \cap B$. Then the derived subgroup of $G_{\alpha}$ is isomorphic to $S L(2)$ or to $P S L(2)$, and $G_{\alpha} / B_{\alpha}$ is isomorphic to the projective line $\mathbb{P}^{1}$. For a $B_{\alpha}$-module $M$, we shall denote the associated $G_{\alpha}$-linearized locally free sheaf on $G_{\alpha} / B_{\alpha}$ by $\underline{M}$.

Notice that $X_{w}, X^{v}$ and hence $X_{w}^{v}$ are invariant under $G_{\alpha}$, and $\left(\partial X_{w}\right)^{v}$ is invariant under $B_{\alpha}$; we have

$$
\left(\partial X_{w}\right)^{v}=X_{s_{\alpha} w}^{v} \cup G_{\alpha}\left(\partial X_{s_{\alpha} w}\right)^{v} .
$$

Consider the fiber product $G_{\alpha} \times{ }^{B_{\alpha}} X_{s_{\alpha} w}^{v}$ with projection

$$
p: G_{\alpha} \times{ }^{B_{\alpha}} X_{s_{\alpha} w}^{v} \longrightarrow G_{\alpha} / B_{\alpha} \simeq \mathbb{P}^{1}
$$

and "multiplication" map

$$
\psi: G_{\alpha} \times{ }^{B_{\alpha}} X_{s_{\alpha} w}^{v} \longrightarrow X_{w}^{v} .
$$

Then $\psi$ is birational (since it is an isomorphism at $e_{w}$ ). Further, we have

$$
\left(\partial X_{w}\right)^{v}=\psi\left(X_{s_{\alpha} w}^{v} \cup G_{\alpha} \times{ }^{B_{\alpha}}\left(\partial X_{s_{\alpha} w}\right)^{v}\right)
$$

where $X_{s_{\alpha} w}^{v}$ is the fiber of $p$ at $B_{\alpha} / B_{\alpha}$. By the projection formula, it follows that

$$
L_{\lambda}\left(-\left(\partial X_{w}\right)^{v}\right)=\psi_{*} \psi^{*} L_{\lambda}\left(-X_{s_{\alpha} w}^{v}-G_{\alpha} \times{ }^{B_{\alpha}}\left(\partial X_{s_{\alpha} w}\right)^{v}\right) .
$$

This yields an isomorphism

$$
H^{0}\left(X_{w}^{v}, L_{\lambda}\left(-\left(\partial X_{w}\right)^{v}\right)\right) \cong H^{0}\left(G_{\alpha} / B_{\alpha}, p_{*} \psi^{*} L_{\lambda}\left(-X_{s_{\alpha} w}^{v}-G_{\alpha} \times{ }^{B_{\alpha}}\left(\partial X_{s_{\alpha} w}\right)^{v}\right)\right) .
$$


Further, we may identify the $G_{\alpha}$-linearized sheaf $\left.p_{*} \psi^{*} L_{\lambda}\left(-G_{\alpha} \times^{B_{\alpha}}\left(\partial X_{s_{\alpha} w}\right)^{v}\right)\right)$ on $G_{\alpha} / B_{\alpha}$, to the sheaf $H^{0}\left(X_{s_{\alpha} w}^{v}, L_{\lambda}\left(-\left(\partial X_{s_{\alpha} w}\right)^{v}\right)\right)$. Therefore, we obtain an exact sequence of $B_{\alpha}$-modules

$$
0 \longrightarrow H^{0}\left(X_{w}^{v}, L_{\lambda}\left(-\left(\partial X_{w}\right)^{v}\right)\right) \longrightarrow H^{0}\left(G_{\alpha} / B_{\alpha}, \frac{H^{0}\left(X_{s_{\alpha} w}^{v}, L_{\lambda}\left(-\left(\partial X_{s_{\alpha} w}\right)^{v}\right)\right)}{\longrightarrow H^{0}\left(X_{s_{\alpha} w}^{v}, L_{\lambda}\left(-\left(\partial X_{s_{\alpha} w}\right)^{v}\right)\right)} \longrightarrow 0,\right.
$$

where the map on the right is the "evaluation" map (its surjectivity follows e.g. from Corollary 1).

Next we analyse the $B_{\alpha}-$ module $H^{0}\left(X_{s_{\alpha} w}^{v}, L_{\lambda}\left(-\left(\partial X_{s_{\alpha} w}\right)^{v}\right)\right)$. By Proposition 8 its weights have multiplicity one; they are among the $-\frac{1}{2}\left(s_{\alpha} w(\lambda)+x(\lambda)\right)$, where $v \leq x \leq s_{\alpha} w$, and the weight $-\frac{1}{2}(w(\lambda)+v(\lambda))-\alpha$ occurs, since $p_{s_{\alpha} w, v} \neq 0$; its $\alpha$-weight (the scalar product with $\check{\alpha}$ ) is -2 .

If $s_{\alpha} v \not \leq s_{\alpha} w$, then the span $M$ of $p_{s_{\alpha} w, v}$ is invariant under $B_{\alpha}$. Thus, the $T$-module $H^{0}\left(G_{\alpha} / B_{\alpha}, \underline{M}\right)$ has weights $-\frac{1}{2}(w(\lambda)+v(\lambda))+(m-1) \alpha, m=0,1,2$, each of them having multiplicity one. Further, the kernel of the evaluation map $H^{0}\left(G_{\alpha} / B_{\alpha}, \underline{M}\right) \longrightarrow M$ contains an element of weight $-\frac{1}{2}(w(\lambda)+v(\lambda))$. By the exact sequence above, this weight occurs in $H^{0}\left(X_{w}^{v}, L_{\lambda}\left(-\left(\partial X_{w}\right)^{v}\right)\right)$; using Proposition 8 again, it follows that $p_{w, v} \neq 0$.

On the other hand, if $s_{\alpha} v \leq s_{\alpha} w$, then Lemma 16 (2) applied to $\left(s_{\alpha} w, v\right)$ yields

$$
p_{s_{\alpha} w, s_{\alpha} v}=e_{\alpha} p_{s_{\alpha} w, v} \neq 0 \text {. }
$$

Hence the span $M$ of $p_{s_{\alpha} w, v}$ and $p_{s_{\alpha} w, s_{\alpha} v}$ is a nontrivial $B_{\alpha}$-module with $\alpha$-weights -2 and 0 (note that $e_{\alpha} p_{s_{\alpha} w, s_{\alpha} v}=0$ by weight considerations). Thus, we have an isomorphism of $B_{\alpha}$-modules

$$
M \cong M_{1} \otimes M_{2},
$$

where $M_{1}$ is a one-dimensional $B_{\alpha}-$ module with $\alpha$-weight -1 , and $M_{2}$ is the standard two-dimensional $G_{\alpha}$-module. It follows that the weights of the $T$-module

$$
H^{0}\left(G_{\alpha} / B_{\alpha}, \underline{M}\right) \cong H^{0}\left(G_{\alpha} / B_{\alpha}, \underline{M_{1}}\right) \otimes M_{2}
$$

are exactly $-\frac{1}{2}(w(\lambda)+v(\lambda))-\alpha,-\frac{1}{2}(w(\lambda)+v(\lambda))+\alpha$ (both of multiplicity one) and $-\frac{1}{2}(w(\lambda)+v(\lambda))$ (of multiplicity two). Thus, the kernel of the evaluation map $H^{0}\left(G_{\alpha} / B_{\alpha}, \underline{M}\right) \longrightarrow M$ contains an element of weight $-\frac{1}{2}(w(\lambda)+v(\lambda))$, and we conclude as above.

Case 2: $\langle v(\lambda), \check{\alpha}\rangle=0$. Then $w>s_{\alpha} w \geq v=s_{\alpha} v$, so that $X_{w}, X^{v}$ and $X_{w}^{v}$ are again invariant under $G_{\alpha}$, whereas $\left(\partial X_{w}\right)^{v}$ is $B_{\alpha}$-invariant. Arguing as in Case 1, we obtain the same relation between $H^{0}\left(X_{w}^{v}, L_{\lambda}\left(-\left(\partial X_{w}\right)^{v}\right)\right)$ and $H^{0}\left(X_{s_{\alpha} w}^{v}, L_{\lambda}\left(-\left(\partial X_{s_{\alpha} w}\right)^{v}\right)\right)$; but now the latter $B_{\alpha}$-module contains the span $M$ of $p_{s_{\alpha} w, v}$, as a $B_{\alpha}$-submodule of $\alpha$-weight -1 . As in Case 1 , it follows that $p_{w, v} \neq 0$. Case 3: $\langle v(\lambda), \check{\alpha}\rangle=-2$. Then $w>s_{\alpha} w \geq v>s_{\alpha} v$, and $X^{v}$ is a double divisor in $X^{s_{\alpha} v}$. Therefore, the pair $\left(s_{\alpha} v, s_{\alpha} w\right)$ is admissible. By the induction hypothesis, we have, $p_{s_{\alpha} v, s_{\alpha} w} \neq 0$. Then Case 1 applies to the pair $\left(s_{\alpha} v, w\right)$ and yields $p_{w, s_{\alpha} v} \neq 0$. Further, $X^{v}$ is a double divisor in $X^{s_{\alpha} v}$. Hence by Lemma16 (2) applied to $\left(w, s_{\alpha} v\right)$, we obtain $p_{w, v} \neq 0$.

Now combining Lemmas 11 16 and 17, we obtain

Proposition 9. Let $v, w \in W^{\lambda}, v \leq w$. Then the pair $(v, w)$ is admissible if and only if $p_{w, v}$ is nonzero. In this case, every chain from $v$ to $w$ is a double chain. 


\section{STANDARD MONOMIALS FOR SUMS OF WEIGHTS OF CLASSICAL TYPE}

In this section, we obtain a standard monomial basis for $H^{0}\left(X_{w}^{v}, L_{\lambda_{1}+\cdots+\lambda_{m}}\right)$, where $X_{w}^{v}$ is a Richardson variety in $G / P$, and $\lambda_{1}, \ldots, \lambda_{m}$ are dominant characters of classical type of $P$ (in the sense of Definition 12).

We begin with the case where $m=1$; we shall need a definition, and a result of Deodhar ([9, Lemmas 4.4 and 4.4') on the Bruhat ordering.

Definition 17. Let $w \in W^{P}$ and let $\lambda$ be a dominant character of $P$. We say that $x \in W^{P}$ is $\lambda$-maximal in $w$ (resp. $\lambda$-minimal on $w$ ) if $x y \leq x$ for any $y \in W_{\lambda}$ such that $x y \in W^{P}$ and $x y \leq w$ (resp. if $x y \geq x$ for any $y \in W_{\lambda}$ such that $x y \in W^{P}$ and $x y \geq w)$.

Lemma 18. Let $w \in W$ and $x \in W^{\lambda}$ such that $x \leq w(\lambda)$ (resp. $x \geq v(\lambda)$ ). Then the set $\left\{y \in W_{\lambda} \mid x y \leq w\right\}$ (resp. $\left\{y \in W_{\lambda} \mid w \leq x y\right\}$ admits a unique maximal (resp. minimal) element.

We shall also need the following consequences of this result.

Lemma 19. (1) Let $w \in W^{P}$ and $x \in W^{\lambda}$ such that $x \leq w(\lambda)$ (resp. $x \geq$ $w(\lambda))$. Then $x \in W / W_{\lambda}$ admits a unique lift $\tilde{x} \in \bar{W}^{P}$ such that $\tilde{x} \overline{i s}$ $\lambda$-maximal in $w$ (resp. $\lambda$-minimal on $w)$.

(2) Let $v \leq w \in W^{P}$, then $v$ is $\lambda$-maximal in $w$ (resp. $w$ is $\lambda$-minimal in $v$ ) if and only if $\left(\partial_{\lambda} X^{v}\right)_{w}=\left(\partial X^{v}\right)_{w}\left(\operatorname{resp} .\left(\partial_{\lambda} X_{w}\right)^{v}=\left(\partial X_{w}\right)^{v}\right)$.

Proof. (1) Let $x \leq w(\lambda)$. By Lemma 18 the set $\left\{y \in W_{\lambda} \mid x y \leq w\right\}$ admits a unique maximal element that we still denote by $y$. Let $\tilde{x}$ be the representative in $W^{P}$ of $x y \in W$, then $\tilde{x}(\lambda)=x y(\lambda)=x(\lambda)$. Further, if we have $\tilde{x} z \leq w$ for some $z \in W_{\lambda}$ such that $\tilde{x} z \in W^{P}$, then we can write $\tilde{x} z=x u$ where $u \in W_{\lambda}$. Since $x u \leq w$, we have $u \leq y$ and hence $x u \leq x y$ (since $x \in W^{\lambda}$ and $u, y \in W_{\lambda}$ ). But $x u=\tilde{x} z \in W^{P}$, so that $\tilde{x} z \leq \tilde{x}$. This proves the assertion concerning $\lambda$-maximal elements, and hence the dual assertion concerning $\lambda$-minimal elements.

(2) If $\left(\partial_{\lambda} X^{v}\right)_{w} \neq\left(\partial X^{v}\right)_{w}$, then there exists $y \in W_{\lambda}$ such that $v<v y \leq w$ and $\ell(v y)=\ell(v)+1$. Thus, $v$ is not $\lambda$-maximal in $w$.

Conversely, if $v$ is not $\lambda$-maximal in $w$, then $v<\tilde{v} \leq w$ where $\tilde{v} \in v W_{\lambda}$ is $\lambda$-maximal in $w$. Hence there exists $y \in W_{\lambda}$ such that $v<v y \leq \tilde{v} \leq w$ and $\ell(v y)=\ell(v)+1$. Now $X_{w}^{v y}$ is contained in $\left(\partial X^{v}\right)_{w}$ but not in $\left(\partial_{\lambda} X^{v}\right)_{w}$.

Now we consider the $T$-module $H^{0}\left(X_{w}^{v}, L_{\lambda}\right)$, where $v \leq w \in W^{P}$ and $\lambda$ is a dominant character of $P$, not necessarily $P$-regular. Notice that the diagram

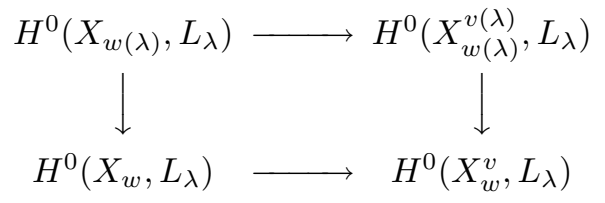

is commutative, where the horizontal (resp. vertical) maps are restrictions (resp. pull-backs). Further, both restrictions are surjective by Proposition 1, and the pull-back on the left is an isomorphism, since the natural map $f: X_{w} \rightarrow X_{w(\lambda)}$ satisfies $f_{*} \mathcal{O}_{X_{w}}=\mathcal{O}_{X_{w(\lambda)}}$. Thus, we may regard the $T$-module $H^{0}\left(X_{w}^{v}, L_{\lambda}\right)$ as a quotient of $H^{0}\left(X_{w(\lambda)}^{v(\lambda)}, L_{\lambda}\right)$. 
Likewise, by using the commutative diagram

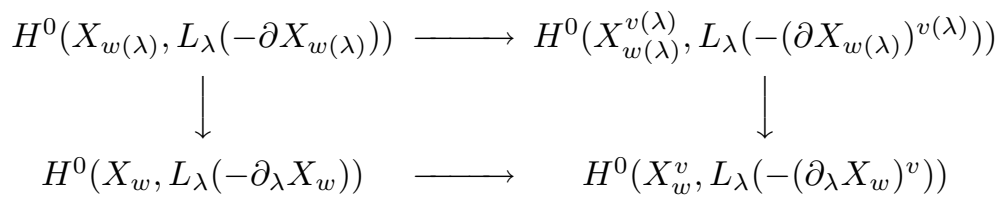

and Corollary 1 we may regard the $T$-module $H^{0}\left(X_{w}^{v}, L_{\lambda}\left(-\left(\partial_{\lambda} X_{w}\right)^{v}\right)\right)$ as a quotient of $H^{0}\left(X_{w(\lambda)}^{v(\lambda)}, L_{\lambda}\left(-\left(\partial X_{w(\lambda)}\right)^{v(\lambda)}\right)\right)$. The latter has been described in Section 6 , in the case that $\lambda$ is of classical type: it has a basis consisting of the $p_{w(\lambda), x(\lambda)}$ where $x(\lambda) \in W^{\lambda}, v(\lambda) \leq x(\lambda) \leq w(\lambda)$ and the pair $(x(\lambda), w(\lambda))$ is admissible. From that description we shall deduce

Proposition 10. Let $v \leq w \in W^{P}$ and let $\lambda$ be a dominant character of classical type of $P$.

(1) The space $H^{0}\left(X_{w}^{v}, L_{\lambda}\left(-\left(\partial_{\lambda} X_{w}\right)^{v}-\left(\partial X^{v}\right)_{w}\right)\right)$ is spanned by $p_{w(\lambda), v(\lambda)}$, if $v$ is $\lambda$-maximal in $w$; otherwise, this space is zero.

(2) The $p_{w(\lambda), x(\lambda)}$ where $x \in W^{P}$ and $v \leq x \leq w$, form a basis of the space $H^{0}\left(X_{w}^{v}, L_{\lambda}\left(-\left(\partial_{\lambda} X_{w}\right)^{v}\right)\right)$.

(3) The $p_{w(\lambda), x(\lambda)}$ where $x \in W^{P}$ is $\lambda$-minimal on $v$, and $w$ is $\lambda$-minimal on $x$, form a basis of $H^{0}\left(X_{w}^{v}, L_{\lambda}\left(-\left(\partial X_{w}\right)^{v}\right)\right)$.

(4) The $p_{y(\lambda), x(\lambda)}$ where $x, y \in W^{P}$ and $v \leq x \leq y \leq w$, form a basis of $H^{0}\left(X_{w}^{v}, L_{\lambda}\right)$.

Proof. (1) Assume that $H^{0}\left(X_{w}^{v}, L_{\lambda}\left(-\left(\partial_{\lambda} X_{w}\right)^{v}-\left(\partial X^{v}\right)_{w}\right)\right)$ contains a nonzero element $p$. Then, by the argument of Proposition $8, \frac{p^{2}}{p_{w(\lambda)} p_{v(\lambda)}}$ is a rational function on $X_{w}^{v}$, without poles; further, it vanishes identically on $\left(\partial X^{v}\right)_{w}-\left(\partial_{\lambda} X^{v}\right)_{w}$, since the zero locus of $p_{v(\lambda)}$ is $\left(\partial_{\lambda} X^{v}\right)_{w}$. It follows that $p^{2}$ is a constant multiple of $p_{w(\lambda)} p_{v(\lambda)}$, and that $\left(\partial X^{v}\right)_{w}=\left(\partial_{\lambda} X^{v}\right)_{w}$. Hence $p$ is a constant multiple of $p_{w(\lambda), v(\lambda)}$, and $v$ is $\lambda$-maximal in $w$ (by Lemma 19).

Conversely, let $v$ be $\lambda$-maximal in $w$; then $\left(\partial_{\lambda} X^{v}\right)_{w}=\left(\partial X^{v}\right)_{w}$. Thus, $p_{w(\lambda), v(\lambda)}$ vanishes identically on $\left(\partial_{\lambda} X_{w}\right)^{v} \cup\left(\partial X^{v}\right)_{w}$. Further, $p_{w(\lambda), v(\lambda)} \neq 0$ on $X_{w}^{v}$, since $p_{w(\lambda), v(\lambda)}^{2}=p_{w(\lambda)} p_{v(\lambda)}$ on $X_{w}$ (by Lemma 12).

(2) By Proposition 8 the space $H^{0}\left(X_{w}^{v}, L_{\lambda}\left(-\left(\partial_{\lambda} X_{w}\right)^{v}\right)\right)$ is spanned by the images of the $p_{w(\lambda), x(\lambda)}$ where $v(\lambda) \leq x(\lambda) \leq w(\lambda)$. Further, $p_{w(\lambda), x(\lambda)}^{2}=p_{w(\lambda)} p_{x(\lambda)}$ on $X_{w}$. Using Lemma 2, we see that $p_{w(\lambda), x(\lambda)}$ is nonzero on $X_{w}^{v}$ if and only if $x(\lambda)$ has a representative $x \in W^{P}$ such that $v \leq x \leq w$.

(3) $H^{0}\left(X_{w}^{v}, L_{\lambda}\left(-\left(\partial X_{w}\right)^{v}\right)\right)$ is a $T$-stable subspace of $H^{0}\left(X_{w}^{v}, L_{\lambda}\left(-\left(\partial_{\lambda} X_{w}\right)^{v}\right)\right)$; thus, it is spanned by certain $p_{w(\lambda), x(\lambda)}$ where $v \leq x \leq w$. By Lemma 12, the zero locus $\left(p_{w(\lambda), x(\lambda)}=0\right)$ in $X_{w}^{v}$ equals $\left(p_{x(\lambda)}=0\right) \cup\left(\partial_{\lambda} X_{w}\right)^{v}$. Hence $p_{w(\lambda), x(\lambda)}$ belongs to $H^{0}\left(X_{w}^{v}, L_{\lambda}\left(-\left(\partial X_{w}\right)^{v}\right)\right)$ if and only if $p_{x(\lambda)}$ vanishes identically on $\left(\partial X_{w}\right)^{v}-$ $\left(\partial_{\lambda} X_{w}\right)^{v}$. By Lemma 2, this amounts to: $x(\lambda)$ admits no lift $x^{\prime}$ such that $v \leq x^{\prime} \leq$ $w y$ for some $y \in W_{\lambda}, w y<y, \ell(w y)=\ell(w)-1$. Let $\tilde{x}$ be the lift of $x(\lambda)$ that is $\lambda$-minimal on $v$, then the preceding condition means that $w$ is $\lambda$-minimal on $\tilde{x}$.

(4) By Proposition [3, we obtain a basis of the space $H^{0}\left(X_{w}^{v}, L_{\lambda}\right)$ by choosing a basis of $H^{0}\left(X_{w}^{x}, L_{\lambda}\left(-\left(\partial X^{x}\right)_{w}\right)\right)$ for each $x \in W^{P}$ such that $v \leq x \leq w$, and lifting this basis to $H^{0}\left(X_{w}^{v}, L_{\lambda}\right)$ under the (surjective) restriction map $H^{0}\left(X_{w}^{v}, L_{\lambda}\right) \rightarrow$ $H^{0}\left(X_{w}^{x}, L_{\lambda}\right)$. Together with (3), it follows that a basis of $H^{0}\left(X_{w}^{v}, L_{\lambda}\right)$ consists of the $p_{y(\lambda), x(\lambda)}$, where $y$ is $\lambda$-maximal in $w, x$ is $\lambda$-maximal in $y$, and $v \leq x$. But 
given any $x^{\prime}, y^{\prime} \in W^{P}$ such that $v \leq x^{\prime} \leq y^{\prime} \leq w$, we have $v \leq x \leq y \leq w$ and $p_{y^{\prime}(\lambda), x^{\prime}(\lambda)}=p_{y(\lambda), x(\lambda)}$, where $x$ (resp. $y$ ) is the representative of $x(\lambda)$ that is $\lambda$-maximal in $w$ (resp. $x$ ).

Definition 18. Let $\lambda_{1}, \ldots, \lambda_{m}$ be dominant characters of classical type of $P$. Let $\pi_{i}=\left(w_{i}, v_{i}\right)$ where $v_{i} \leq w_{i} \in W^{\lambda_{i}}$ for $1 \leq i \leq m$. Then the sequence $\underline{\pi}=$ $\left(\pi_{1}, \ldots, \pi_{m}\right)$ is standard if there exist lifts $\tilde{w}_{i}, \tilde{v}_{i}$ in $W^{P}$ for $1 \leq i \leq m$, such that

$$
\tilde{v}_{m} \leq \tilde{w}_{m} \leq \cdots \leq \tilde{v}_{1} \leq \tilde{w}_{1} .
$$

The monomial

$$
p_{\underline{\pi}}=p_{w_{1}\left(\lambda_{1}\right), v_{1}\left(\lambda_{1}\right)} \cdots p_{w_{m}\left(\lambda_{m}\right), v_{m}\left(\lambda_{m}\right)} \in H^{0}\left(G / P, L_{\lambda_{1}+\cdots+\lambda_{m}}\right)
$$

is called standard as well.

Further, let $v, w \in W^{P}$ such that $v \leq w$; then $\underline{\pi}$ is standard on $X_{w}^{v}$ if there exist lifts as above, such that

$$
v \leq \tilde{v}_{m} \leq \tilde{w}_{m} \leq \cdots \leq \tilde{v}_{1} \leq \tilde{w}_{1} \leq w .
$$

The restriction of $p_{\underline{\pi}}$ to $X_{w}^{v}$ is called a standard monomial on $X_{w}^{v}$; it is a $T$ eigenvector in $H^{0}\left(X_{w}^{\bar{v}}, L_{\lambda_{1}+\cdots+\lambda_{m}}\right)$.

Notice that there is no loss of generality in assuming that $\tilde{v}_{m}$ is $\lambda_{m}$-minimal on $v$, and that $\tilde{w}_{m}$ is $\lambda_{m}$-minimal on $\tilde{v}_{m}$.

Now the argument of Proposition 7 together with Proposition 10 and induction on $m$, yields the following partial generalization of Corollary 3

Theorem 3. Let $v \leq w \in W^{P}$ and let $\lambda_{1}, \ldots, \lambda_{m}$ be dominant characters of $P$. If $\lambda_{1}, \ldots, \lambda_{m}$ are of classical type, then the standard monomials on $X_{w}^{v}$ form a basis for $H^{0}\left(X_{w}^{v}, L_{\lambda_{1}+\cdots+\lambda_{m}}\right)$.

Remarks. (1) In particular, Theorem 3 applies to $P=B$ if all fundamental weights are of classical type, that is, if $G$ is classical. Thereby, we retrieve all results of [9].

(2) The second assertion of Corollary 3 does not generalize to this setting; that is, there are examples of standard monomials on $G / P$ which are not standard on $X_{w}^{v}$, but which restrict nontrivially to that subvariety.

Specifically, let $G=S L(3)$ with simple reflections $s_{1}, s_{2}$ and fundamental weights $\omega_{1}, \omega_{2}$. Then one may check that the monomial

$$
p_{s_{1}\left(\omega_{1}\right)} p_{s_{2}\left(\omega_{2}\right)} \in H^{0}\left(G / B, L_{\omega_{1}+\omega_{2}}\right)
$$

is standard on $G / B$ and restricts nontrivially to $X_{s_{2} s_{1}}$, but is not standard there.

\section{REFERENCES}

[1] M. Brion: Positivity in the Grothendieck group of complex flag varieties, J. Algebra 258 (2002), 137-159. MR 2003m:14017

[2] M. BRion and P. Polo: Large Schubert varieties, Represent. Theory 4 (2000), 97-126. MR 2001j:14066

[3] C. Chevalley: Sur les décompositions cellulaires des espaces $G / B$ (with a foreword by A. Borel), Proc. Sympos. Pure Math. 56, Part 1, Algebraic Groups and their Generalizations: Classical Methods (University Park, PA; 1991), Amer. Math. Soc., Providence, RI (1994), 1-23. MR 95e:14041

[4] V. V. Deodhar: On some geometric aspects of Bruhat orderings. I. A finer decomposition of Bruhat cells. Invent. Math. 79 (1985), 499-511. MR 86f:20045 
[5] W. V. D. Hodge: Some enumerative results in the theory of forms, Proc. Camb. Phil. Soc. 39 (1943), 22-30. MR 4:184e

[6] B. Kostant and S. Kumar: $T$-equivariant $K$-theory of generalized flag varieties, J. Differential Geom. 32 (1990), 549-603. MR 92c:19006

[7] A. Knutson: A Littelmann-type formula for Duistermaat-Heckman measures, Invent. Math. 135 (1999), 185-200. MR 2000d:53127

[8] V. Lakshmibai and P. Littelmann: Richardson varieties and equivariant $K$-theory, J. Algebra 260 (2003), 230-260.

[9] V. Lakshmibai and C.S. Seshadri: Geometry of G/P-V, J. Alg, 100 (1986), 462-557. MR 87k:14059

[10] V. Lakshmibai and C.S. Seshadri: Standard monomial theory, Proc. Hyderabad Conference on Algebraic Groups, (S. Ramanan et al., eds.), Manoj Prakashan, Madras (1991), 279-322. MR 92k:14053

[11] P. Littelmann: A Littlewood-Richardson rule for symmetrizable Kac-Moody algebras, Invent. Math. 116 (1994), 329-346. MR 95f:17023

[12] P. Littelmann: Contracting modules and standard monomial theory, J. Amer. Math. Soc. 11 (1998), 551-567. MR 99d:17027

[13] V. Mehta and W. van DeR Kallen: On a Grauert-Riemenschneider theorem for Frobenius split varieties in characteristic $p$, Invent. Math. 108 (1992), 11-13. MR 93a:14017

[14] S. Ramanan and A. Ramanathan: Projective normality of flag varieties and Schubert varieties, Invent. Math. 79 (1985), 217-234. MR 86j:14051

[15] A. Ramanathan: Schubert varieties are arithmetically Cohen-Macaulay, Invent. Math. 80 (1985), 283-294. MR 87d:14044

[16] A. Ramanathan: Equations defining Schubert varieties and Frobenius splitting of diagonals, Pub. Math. IHES 65 (1987), 61-90. MR 88k:14032

[17] R. W. Richardson: Intersections of double cosets in algebraic groups, Indag. Math. (N. S.) 3 (1992), 69-77. MR 93b:20081

Institut Fourier, UMR 5582 du CNRS, F-38402 SAint-Martin D'Hères Cedex

E-mail address: Michel.Brion@ujf-grenoble.fr

Department of Mathematics, Northeastern University, Boston, Massachusetts 021155096

E-mail address: lakshmibai@neu.edu 